\title{
Completeness and Nonclassicality of Coherent States for Generalized Oscillator Algebras
}

\author{
Kevin Zelaya, ${ }^{1}$ Oscar Rosas-Ortiz, ${ }^{1}$ Zurika Blanco-Garcia, ${ }^{1}$ and Sara Cruz y Cruz ${ }^{2}$ \\ ${ }^{1}$ Physics Department, Cinvestav, AP 14-740, 07000 Mexico City, Mexico \\ ${ }^{2}$ Instituto Politécnico Nacional, UPIITA, Av. I.P.N. 2580, Col. La Laguna Ticomán, 07360 Mexico City, Mexico \\ Correspondence should be addressed to Oscar Rosas-Ortiz; orosas@fis.cinvestav.mx
}

Received 2 August 2017; Revised 10 November 2017; Accepted 19 November 2017; Published 25 December 2017

Academic Editor: Claudio Dappiaggi

Copyright (c) 2017 Kevin Zelaya et al. This is an open access article distributed under the Creative Commons Attribution License, which permits unrestricted use, distribution, and reproduction in any medium, provided the original work is properly cited.

\begin{abstract}
The purposes of this work are (1) to show that the appropriate generalizations of the oscillator algebra permit the construction of a wide set of nonlinear coherent states in unified form and (2) to clarify the likely contradiction between the nonclassical properties of such nonlinear coherent states and the possibility of finding a classical analog for them since they are $P$-represented by a delta function. In (1) we prove that a class of nonlinear coherent states can be constructed to satisfy a closure relation that is expressed uniquely in terms of the Meijer $G$-function. This property automatically defines the delta distribution as the $P$-representation of such states. Then, in principle, there must be a classical analog for them. Among other examples, we construct a family of nonlinear coherent states for a representation of the $\mathrm{su}(1,1)$ Lie algebra that is realized as a deformation of the oscillator algebra. In (2), we use a beam splitter to show that the nonlinear coherent states exhibit properties like antibunching that prohibit a classical description for them. We also show that these states lack second-order coherence. That is, although the $P$-representation of the nonlinear coherent states is a delta function, they are not full coherent. Therefore, the systems associated with the generalized oscillator algebras cannot be considered "classical" in the context of the quantum theory of optical coherence.
\end{abstract}

\section{Introduction}

The nonclassical properties of light have received a great deal of attention in recent years, mainly in connection with quantum optics [1], quantum information [2], and the principles of quantum mechanics [3]. Among other nonclassical profiles, the related states can be generated by nonlinear processes to have sub-Poissonian statistics [4] or to exhibit squeezing (reduction of the variance) in one quadrature [5]. Some deformations of the algebra generated by the conventional boson operators have been proposed to represent photons with "unusual properties" [6, 7], which have found applications in the photon counting statistics, squeezing, and signalto-quantum noise ratio [8]. The immediate generalizations $[9,10]$ motivated the development of the subject as an important branch of quantum optics [1]. The deformations of the boson algebra include supersymmetric structures [1114] for which the so-called polynomial Heisenberg algebras are quite natural [15-18]. Recently, some non-Hermitian models have been shown to obey the distortions of the boson algebra that arise in the conventional supersymmetric approaches $[19,20]$. In all cases, the deformed oscillator algebras have been used to construct the corresponding generalized (also called nonlinear) coherent states. Most of these states exhibit nonclassical properties that distinguish them from the coherent states of the conventional boson algebra.

In this work we propose a modification of the conventional boson (also called oscillator) algebra that permits the recovering of the majority of the already studied deformed boson algebras as particular cases. The nonlinear coherent states of these generalized oscillator algebras can be written in the same mathematical form, which facilitates their study. We shall address the discussion to polynomial-like algebras because, as we are going to prove, the closure relation satisfied by the corresponding nonlinear coherent states is expressed uniquely in terms of the Meijer G-function. Accordingly, the $P$-representation $[21,22]$ of all the nonlinear coherent states we deal with is as singular as the $\delta$-distribution. 
Then, following $[23,24]$, the fields represented by such states would have a classical analog. However, these states have nonclassical properties that can be exhibited either with the help of a beam splitter [25] (see also [26]) or by showing that their statistics is sub-Poissonian [4]. We face this likely contradiction by interpreting the action of a beam splitter on a given photon state as the equivalent of a double-slit interference experiment in the single-photon regime [27, 28] (see also [29]). Then, we use this equivalence to show that the fields represented by the nonlinear coherent states exhibit antibunching (the probability of two or more photons arriving together at the same point is zero), so they cannot be modeled in classical form. The conclusion is that the nonlinear coherent states are not full coherent although their $P$-representation is a delta function. Then, they do not satisfy the notion of classicalness introduced by Glauber in his quantum theory of optical coherence [23].

The paper is organized as follows. In Section 2 we generalize the oscillator algebra and give some immediate examples. Section 3 deals with the construction of the nonlinear coherent states. We show that the completeness of these states is always possible for the polynomial-like algebras in terms of the Meijer $G$-function. Some examples are also discussed. In Section 4 we show that the $P$-representation of the nonlinear coherent states is given by the $\delta$-distribution while the photon states are represented by the derivatives of $\delta$. In Section 5 we analyze the likely contradiction between the completeness and the nonclassicality of the nonlinear coherent states. Some final remarks are given in Section 6. We have added an appendix with some important mathematical expressions that are not required for reading the paper but are necessary to follow the calculations.

\section{Generalized Oscillators}

The conventional boson ladder operators $\hat{a}$ and $\hat{a}^{\dagger}$ satisfy the algebraic relation $\left[\hat{a}, \hat{a}^{\dagger}\right]=1$. If the number operator $\widehat{n}=\widehat{a}^{\dagger} \widehat{a}$ is considered, then $[\widehat{n}, \widehat{a}]=-\widehat{a}$ and $\left[\widehat{n}, \hat{a}^{\dagger}\right]=\widehat{a}^{\dagger}$. The latter expressions define the oscillator (or boson) algebra and show that the action of $\hat{a}^{\dagger}$ on any number eigenvector $|n\rangle, n=0,1,2, \ldots$, produces a new eigenvector of $\hat{n}$ with eigenvalue $n+1$. Similarly, $\widehat{a}|n+1\rangle$ is proportional to the number eigenvector $|n\rangle$, while $\hat{a}|0\rangle=0$. In the sequel we shall modify the oscillator algebra by preserving the number operator $\hat{n}$ but changing the ladder operators, now written $\widehat{a}_{E}$ and $\widehat{a}_{E}^{\dagger}$, as the set of generators. We say that any system obeying the new algebra is a generalized oscillator.

2.1. Deformed Oscillator Algebras. To define the generalized oscillators let us introduce the pair of ladder operators

$$
\begin{aligned}
& \widehat{a}_{E}|n\rangle=\sqrt{E(n)}|n-1\rangle, \\
& \hat{a}_{E}^{\dagger}|n\rangle=\sqrt{E(n+1)}|n+1\rangle,
\end{aligned}
$$

$$
n=0,1,2, \ldots,
$$

where $E$ is a nonnegative function, $\widehat{a}_{E}^{\dagger}$ is the Hermitian conjugate of $\widehat{a}_{E}$, and $|n\rangle$ is eigenvector of the number operator with eigenvalue $n$. The following algebraic relations can be proven:

$$
\begin{aligned}
& {\left[\widehat{n}, \widehat{a}_{E}\right]=-\widehat{a}_{E},} \\
& {\left[\widehat{n}, \hat{a}_{E}^{\dagger}\right]=\widehat{a}_{E}^{\dagger} .}
\end{aligned}
$$

The product $\widehat{a}_{E}^{\dagger} \widehat{a}_{E}$ preserves the number of quanta since it is equal to the function $E(\widehat{n})$. Equivalently, $\widehat{a}_{E} \widehat{a}_{E}^{\dagger}=E(\widehat{n}+1)$. Then

$$
\left[\widehat{a}_{E}, \widehat{a}_{E}^{\dagger}\right]=E(\widehat{n}+1)-E(\widehat{n}) .
$$

As the vacuum state $|0\rangle$ does not contain quanta we shall assume $E(0)=0$ in order to have $\widehat{a}_{E}|0\rangle=0$. The number eigenvectors

$$
|n\rangle=\frac{\left(a^{\dagger}\right)^{n}}{\sqrt{n !}}|0\rangle, \quad n=0,1,2, \ldots,
$$

are now expressed as

$$
\begin{aligned}
& |n\rangle=\frac{\left(a_{E}^{\dagger}\right)^{n}}{\sqrt{E(n) !}}|0\rangle, \\
& E(n) !=E(1) E(2) \cdots E(n), E(0) ! \equiv 1, n=0,1, \ldots,
\end{aligned}
$$

and will be used as the orthonormal basis of the Hilbert space $\mathscr{H}$. The latter consists of all vectors

$$
\begin{aligned}
|\psi\rangle & =\sum_{n=0}^{\infty} \psi_{n}|n\rangle, \\
\psi_{n} & :=\langle n \mid \psi\rangle \in \mathbb{C},
\end{aligned}
$$

such that

$$
\langle\psi \mid \psi\rangle=\sum_{n=0}^{\infty}\left|\psi_{n}\right|^{2}<\infty
$$

2.2. Examples. The following list of examples does not exhaust all the possible generalizations of the oscillator algebras that can be performed with the rules defined in (1)-(3). We explicitly mention such cases because either they are connected with approaches already reported, or they give rise to very important results in mathematical physics or they find applications in quantum optics.

2.2.1. $f$-Oscillators. An important class of generalized harmonic oscillators has been already introduced and is nowadays known as the set of $f$-oscillators [10]. These oscillators are recovered here by making $E(\widehat{n})=\widehat{n} f^{2}(\widehat{n})$ in (1)-(3), with $f$ a properly chosen real-valued function. In this case the ladder operators $\widehat{a}_{E}$ and $\hat{a}_{E}^{\dagger}$ are factorized as

$$
\begin{aligned}
& \widehat{a}_{E}=\widehat{a} f(\widehat{n})=f(\widehat{n}+1) \hat{a}, \\
& \widehat{a}_{E}^{\dagger}=f(\widehat{n}) \hat{a}^{\dagger}=\widehat{a}^{\dagger} f(\widehat{n}+1) .
\end{aligned}
$$

The $f$-oscillators have been associated with the center-ofmass motion of a trapped and bichromatically laser-driven ion [9] and are also related to a "frequency blue shift" in high intensity photon beams [10]. 
2.2.2. q-Deformed Oscillators. It has been shown [10] that the special choice of the $f$-function

$$
f(\widehat{n})=\sqrt{\frac{\sinh (\lambda \widehat{n})}{\widehat{n} \sinh \lambda}}, \quad \lambda=\ln q, q \in \mathbb{R},
$$

reduces commutator (3) of the $f$-operators (8) to the rule of $q$-deformed oscillators obeyed by the "physics" bosons $[6,7]$

$$
\widehat{a}_{E} \widehat{a}_{E}^{\dagger}-q \widehat{a}_{E}^{\dagger} \widehat{a}_{E}=q^{-\widehat{n}} .
$$

The above approach has important applications in quantum optics as regards the photon counting statistics, squeezing and signal-to-quantum noise ratio [8].

2.2.3. Polynomial-Like Oscillators. Other relevant classes of oscillators are obtained by assuming that $E(\widehat{n})$ is a real polynomial of degree $\ell \geq 1$,

$$
\begin{aligned}
E(\widehat{n}) & =\prod_{p=1}^{\ell}\left(\alpha_{p} \widehat{n}+\beta_{p}\right)=\gamma_{\ell} \prod_{p=1}^{\ell}\left(\widehat{n}+\delta_{p}\right), \\
\gamma_{\ell} & =\prod_{p=1}^{\ell} \alpha_{p}, \\
\delta_{p} & =\frac{\beta_{p}}{\alpha_{p}} .
\end{aligned}
$$

The expression

$$
E(n) !=\gamma_{\ell}^{n} \prod_{p=1}^{\ell} \frac{\Gamma\left(n+1+\delta_{p}\right)}{\Gamma\left(1+\delta_{p}\right)}
$$

is easily achieved and will be useful in the sequel. In this case the commutator relations (2)-(3) define a polynomial Heisenberg algebra of degree $\ell-1$. The polynomial algebras are quite natural in the higher order supersymmetric approaches [11-14] and are usually connected with nonlinearities that arise because the differential order of the operators that intertwine the SUSY partner Hamiltonians is greater than one [12]. The examples discussed in Sections 2.2.4, 2.2.5, and 2.2.6 are special cases of polynomial algebras.

(i) The simplest example is obtained for $\ell=1$. The commutator (3) is the 0 -degree polynomial $E(\widehat{n}+1)-$ $E(\widehat{n})=\alpha_{1}$. The condition $E(0)=0$ implies $\beta_{1}=0$, by necessity. Then, for $\alpha_{1}=1$ one gets $E(\widehat{n})=$ id, with id $\equiv \rrbracket$ the identity operator in $\mathscr{H}$. In this case $\widehat{a}_{\text {id }}=\widehat{a}$ and $\hat{a}_{\mathrm{id}}^{\dagger}=\widehat{a}^{\dagger}$, so that (2)-(3) define the conventional algebra of the oscillator.

2.2.4. $s u(1,1)$-Oscillators. If $E$ is a quadratic polynomial $(\ell=$ 2 ), the commutator (3) gives rise to the first-order degree polynomial

$$
E(\widehat{n}+1)-E(\widehat{n})=2 \alpha_{1} \alpha_{2} \widehat{n}+\alpha_{1} \beta_{2}+\alpha_{2} \beta_{1}+\alpha_{1} \alpha_{2} .
$$

A striking example occurs for $\beta_{2}=0$ since the related function $E(\widehat{n})=\alpha_{1} \alpha_{2} \widehat{n}^{2}+\alpha_{2} \beta_{1} \widehat{n}$ leads to the $\operatorname{su}(1,1)$ Lie algebra

$$
\begin{aligned}
& {\left[K_{0}, K_{ \pm}\right]= \pm K_{ \pm},} \\
& {\left[K_{-}, K_{+}\right]=2 K_{0}}
\end{aligned}
$$

(similar expressions are obtained for $\beta_{1}=0$ and arbitrary $\left.\beta_{2}\right)$. Indeed, by making $E(\widehat{n})=\widehat{n} f^{2}(\widehat{n})$ with $f(\widehat{n})=$ $\sqrt{\alpha_{2}\left(\alpha_{1} \hat{n}+\beta_{1}\right)}$, the identification

$$
\begin{aligned}
& K_{-}=\widehat{a}_{E}=\widehat{a} \sqrt{\alpha_{2}\left(\alpha_{1} \widehat{n}+\beta_{1}\right)}, \\
& K_{+}=\widehat{a}_{E}^{\dagger}=\sqrt{\alpha_{2}\left(\alpha_{1} \hat{n}+\beta_{1}\right)} \hat{a}^{\dagger}, \\
& K_{0}=\alpha_{1} \alpha_{2} \hat{n}+\frac{1}{2}\left(\alpha_{2} \beta_{1}+\alpha_{1} \alpha_{2}\right)
\end{aligned}
$$

gives the algebra (14) from relations (2)-(3).

(ii) If additionally to $\beta_{2}=0$ we take $\alpha_{1}=\alpha_{2}=$ $\beta_{1}=1$, then $E(\widehat{n})=\widehat{n}(\widehat{n}+1)$. The generators of the $\operatorname{su}(1,1)$ Lie algebra are in this case $K_{0}=\hat{n}+$ $1, K_{-}=\widehat{a} \sqrt{\hat{n}+1}$, and $K_{+}=K_{-}^{\dagger}$. The operators $K_{ \pm}$have been already used to represent the atomphoton coupling of the Jaynes-Cummings model [30] for intensity dependent interactions [31,32].

2.2.5. SUSY-Like Oscillators. A special case of commutator (3) is obtained if the $E$-function is a cubic polynomial $(\ell=3)$ such that $E(1)=E(0)=0$. The latter condition means that the vacuum $|0\rangle$ is annihilated by both ladder operators, $\widehat{a}_{E}$ and $\widehat{a}_{E}^{\dagger}$, while the 1 -photon state $|1\rangle$ is annihilated by $\widehat{a}_{E}$. That is,

$$
\widehat{a}_{E}|1\rangle=\widehat{a}_{E}|0\rangle=\widehat{a}_{E}^{\dagger}|0\rangle=0 .
$$

Then, the action of the ladder operators on the subspace $\{\mid n+$ $2\rangle, n=0,1,2, \ldots\}$ is a modification of rule (1); namely,

$$
\begin{aligned}
& \widehat{a}_{E}|n+2\rangle=\sqrt{E(n+2)}|n+1\rangle, \\
& \widehat{a}_{E}^{\dagger}|n+1\rangle=\sqrt{E(n+2)}|n+2\rangle,
\end{aligned}
$$

$$
n \geq 0 .
$$

Assuming that the solutions of (16) are given, the number eigenvectors $|n+2\rangle$ are now generated from the 1-photon state

$$
|n+2\rangle=\frac{\widehat{a}_{E}^{\dagger(n+1)}}{\sqrt{E(n+2) !}}|1\rangle, \quad n \geq 0 .
$$

The above construction is associated with the eigenstates of a series of Hermitian Hamiltonians that share their spectrum with the conventional harmonic oscillator, which is shifted in one unit of energy. That is, the energies are given by $E_{n}=n-1 / 2$ [15]. Such Hamiltonians are supersymmetric (SUSY) partners of the conventional oscillator for which the supersymmetry is unbroken [11]. A more general treatment 
includes non-Hermitian Hamiltonians whose eigenvalues are given by $E_{n+1}=n+1 / 2$ and $E_{0}=\epsilon<1 / 2[19,20]$. Thus, the spectrum of such non-Hermitian Hamiltonians includes all the oscillator energies $n+1 / 2$ plus an additional real eigenvalue $\epsilon$ which is located below the ground state energy of the conventional oscillator. The $E$-function for this case can be derived from (11) with $\alpha_{1}=\alpha_{2}=\alpha_{3}=\beta_{1}=1, \beta_{2}=1 / 2-\epsilon$, and $\beta_{3}=3 / 2-\epsilon$. Namely,

$$
E(\widehat{n}+2)=(\widehat{n}+1)\left(\widehat{n}+\frac{1}{2}-\epsilon\right)\left(\widehat{n}+\frac{3}{2}-\epsilon\right), \quad n \geq 0 .
$$

The non-Hermitian Hamiltonians associated with (19) are constructed with a complex-valued potential [19], so that their study requires a biorthogonal structure for the space of states [20]. In the appropriate limit, the imaginary part of the potential is cancelled and the model becomes Hermitian, although the generalized algebra defined by (19) is preserved. Then, in such limit, the algebraic structure of the oscillators reported in [15] is recovered by making $\epsilon=-1 / 2$.

Notice that the algebras described above depend on the ground energy of the system. That is, systems with different ground energies $\epsilon$ will be regulated by different algebras.

2.2.6. Distorted SUSY-Like Oscillators. Another E-function that satisfies the supersymmetric relations (16)-(18) is defined as $E(\widehat{n}+2)=w+\widehat{n}$, with $w$ a nonnegative parameter and $E(1)=E(0)=0$. In this case the commutator (3) gives

$$
E(\widehat{n}+1)-E(\widehat{n})=\left\{\begin{array}{cc}
0 & n=0 \\
w & n=1 \\
1 & n \geq 2 .
\end{array}\right.
$$

Thus, the operators $\widehat{a}_{E}$ and $\widehat{a}_{E}^{\dagger}$ are the generators of an algebra that imitates the Heisenberg one. If we concentrate on the subspace spanned by $\{|n\rangle, n \geq 2\}$ only, then the commutator (20) is completely equivalent to the oscillator one. The same occurs in the subspace spanned by $|1\rangle$, up to the constant $w \geq$ 0 . For this reason, the algebra defined by (20) is referred to as distorted Heisenberg algebra, and $w$ is called the distortion parameter $[16,17]$.

We would like to remark that, contrary to what happens with the algebra of the previous section, the distorted Heisenberg algebra does not depend on the ground energy of the system. Besides, the results associated with (20) are also easily extended to non-Hermitian Hamiltonians by using the biorthogonal approach developed in [20].

Other versions of polynomial algebras can be obtained from either the $\mathcal{N}$-fold or the nonlinear supersymmetric models discussed in $[13,14]$ and [12], respectively.

\section{Nonlinear Coherent States}

Up to a normalization constant, the solutions to the eigenvalue equation $\widehat{a}_{E}\left|z_{E}\right\rangle=z\left|z_{E}\right\rangle$, with $z \in \mathbb{C}$, can be written in the form

$$
\left|z_{E}\right\rangle=\sum_{n=0}^{\infty} \frac{z^{n}}{\sqrt{E(n) !}}|n\rangle
$$

The vector $\left|z_{E}\right\rangle$ belongs to $\mathscr{H}$ only if the series (21) is norm convergent. Thus, if $\left|z_{E}\right\rangle_{N}=\mathcal{N}_{E}(|z|)\left|z_{E}\right\rangle$ denotes the normalized solution ${ }_{N}\left\langle z_{E} \mid z_{E}\right\rangle_{N}=\left|\mathcal{N}_{E}(|z|)\right|^{2}\left\langle z_{E} \mid z_{E}\right\rangle=1$, then the expression

$$
\mathcal{N}_{E}(|z|)=\left[\sum_{n=0}^{\infty} \frac{|z|^{2 n}}{E(n) !}\right]^{-1 / 2}
$$

must be finite. Clearly, not any $E$ and $|z|$ are allowed. In the following we assume that $\mathcal{N}_{E}(|z|)$ is finite for $z \in \mathcal{S} \subseteq \mathbb{C}$, with $\mathcal{S}$ defined whenever $E$ has been provided, and say that $\left|z_{E}\right\rangle_{N}$ is a generalized (nonlinear) coherent state. Concrete realizations will be shown in the examples.

The probability of having $n$ photons associated with the nonlinear coherent state $\left|z_{E}\right\rangle_{N}$ is given by $\mathscr{P}_{E}(n,|z|)=\mid\langle n|$ $\left.z_{E}\right\rangle\left._{N}\right|^{2}=\mathcal{N}_{E}^{2}(|z|)|z|^{2 n} / E(n) !$, and the average photon number is

$$
\langle\widehat{n}\rangle_{z_{E}}=\mathscr{N}_{E}^{2}(|z|) \sum_{n=0}^{\infty} \frac{|z|^{2(n+1)}}{E(n+1) !}(n+1) .
$$

Following [33] we may introduce the $E$-exponential function (see details in Appendix)

$$
e_{E}^{x}=\sum_{n=0}^{\infty} \frac{x^{n}}{E(n) !} .
$$

Then, using (5), the coherent state (21) and the normalization constant (22) can be written in simpler form, respectively, $\left|z_{E}\right\rangle=e_{E}^{z a_{E}^{\dagger}}|0\rangle$ and $\mathcal{N}_{E}(z)=\left(e_{E}^{|z|^{2}}\right)^{-1 / 2}$.

(iii) For the identity function $E=$ id we have $e_{\mathrm{id}}^{x}=e^{x}$ as an appropriate limit; see (A.5) of Appendix. Then, the vectors $\left|z_{\text {id }}\right\rangle_{N} \equiv|z\rangle_{N}$ represent the conventional coherent states of the harmonic oscillator.

3.1. Completeness. As usual, although the nonlinear coherent states $\left|z_{E}\right\rangle_{N}$ are not mutually orthogonal $\left\langle z_{E} \mid z_{E}^{\prime}\right\rangle=$ $\mathcal{N}_{E}(z) \mathcal{N}_{E}\left(z^{\prime}\right) e_{E}^{z^{*} z^{\prime}}$ (hereafter the symbol $*$ denotes complex conjugation), they satisfy a closure relation

$$
\square=\int \mathcal{N}_{E}^{2}(z)\left|z_{E}\right\rangle\left\langle z_{E}\right| d \mu_{E}(z),
$$

with $d \mu_{E}(z)$ a measure function to be determined. Let us write

$$
d \sigma_{E}(z)=\mathcal{N}_{E}^{2}(z) d \mu_{E}(z)=\frac{d^{2} z}{\pi} \Lambda_{E}\left(|z|^{2}\right),
$$

where $\Lambda_{E}$ is an additional function to be determined, $d^{2} z=$ $r d r d \theta$, and $z=r e^{i \theta}$. After integrating over $\theta$, expression (25) is as follows:

$$
\llbracket=\sum_{n=0}^{\infty} \frac{|n\rangle\langle n|}{E(n) !} \int_{0}^{\infty} \Lambda_{E}(x) x^{n} d x, \quad x=r^{2} .
$$

As the number eigenvectors $|n\rangle$ form a complete set, the above equality is achieved whenever $\Lambda_{E}$ satisfies

$$
\int_{0}^{\infty} \Lambda_{E}(x) x^{n} d x=E(n) !
$$

After the change $n \rightarrow m-1$, integral equation (28) coincides with the Mellin transform [34] of $\Lambda_{E}(x)$. 
3.2. Examples. Once algebras (2)-(3) and the related coherent states (21), with closure relation (25), have been given in general form, it is profitable to analyze concrete realizations in detail. The coherent states for the $f$-oscillators of Section 2.2.1 and those for the q-oscillators of Section 2.2.2 have been exhaustively studied in [10] and $[6,7]$, respectively. Hence, it is not necessary to revisit them in the present work. Nevertheless, we would like to mention that the former have been used in approaching the Jaynes-Cummings model for some nonlinear Kerr media [35] and that the coherent states of the $q$-oscillators can be recovered from those reported in [10] as a particular case (see $[36,37]$ for early constructions using other definition of the $E$-function). Next, we pay attention to the coherent states associated with the polynomial algebras derived in Section 2.2.3. The reason is that such algebras are general enough to include a plenty of cases which have a common property. Namely, the measure permitting the resolution of the identity (25) is given in terms of a Meijer G-function.

3.2.1. Polynomial-Like Oscillators. The explicit form of the $E$ exponential function (24) for the $\ell$-polynomial $E$-functions (11)-(12) is given in (A.2) of Appendix. Except for some atypical cases, the normalization constant (22) is well defined so that the related coherent states $\left|z_{E}\right\rangle_{N}$ are in the Hilbert space $\mathscr{H}$. To satisfy closure relation (25), in this case the Mellin transform (28) is simplified by using the change of variables:

$$
\begin{aligned}
y & =\frac{x}{\gamma_{\ell}} \\
M_{E}(y) & =\left[\prod_{p=1}^{\ell} \Gamma\left(1+\delta_{p}\right)\right] \gamma_{\ell} \Lambda_{E}(x) .
\end{aligned}
$$

Thus, we arrive at the moment problem

$$
\int_{0}^{\infty} M_{E}(y) y^{n-1} d y=\Gamma\left(n+\delta_{1}\right) \cdots \Gamma\left(n+\delta_{\ell}\right),
$$

which is the Mellin-Barnes integral representation [38] of the following Meijer G-function:

$$
M_{E}(y)=G_{0, \ell}^{\ell, 0}\left(\begin{array}{l|c}
y & - \\
\delta_{1}, \ldots, \delta_{\ell}
\end{array}\right) .
$$

After substituting this last result into (29) and (26) we obtain the explicit form of the measure we are looking for.

(iv) For $\ell=1$, the introduction of $E(n)=\alpha n+\beta$ in (21)(22) gives the normalized vectors

$$
\begin{aligned}
& \left|z_{E}\right\rangle_{N} \\
& =\left[\frac{\Gamma(1+\delta)}{{ }_{1} F_{1}\left(1,1+\delta,|z|^{2} / \alpha\right)}\right]^{1 / 2} \sum_{n=0}^{\infty} \frac{(z / \sqrt{\alpha})^{n}}{\sqrt{\Gamma(n+1+\delta)}}|z\rangle,
\end{aligned}
$$

where we have used (A.4) of Appendix. The Meijer G-function (31) is very simple in this case $G_{0,1}^{1,0}(x, \delta)=$ $e^{-x} x^{\delta}$. Then, the nonlinear coherent states (32) form an overcomplete set in the Hilbert space $\mathscr{H}$ whenever $E(0)=0$; see paragraph between (3) and (5). Then $\alpha=1$ and $\beta=0$ (equivalently $\delta=0$ ), so that (32) is reduced to the expression of the conventional coherent states $\left|z_{\text {id }}\right\rangle_{N}$.

3.2.2. $s u(1,1)$-Oscillators. For the $E$-function derived in Section 2.2.4, the explicit form of $e_{E}^{x}$ is given in (A.6) of Appendix. In particular, using $\alpha_{1}=\alpha_{2}=\beta_{1}=1$ one gets the expression $e_{E}^{x}=I_{1}(2 \sqrt{x}) / \sqrt{x}$, with $I_{\nu}(z)$ the modified Bessel function of the first kind [38]. Then, the normalization constant $\mathcal{N}_{E}(z)=\sqrt{|z| / I_{1}(2|z|)}$ is finite for any $z \in \mathbb{C}$, and the measure acquires the form

$$
\begin{aligned}
d \mu_{E}(z) & =\frac{d^{2} z}{\pi} \frac{I_{1}(2|z|)}{|z|} G_{0,2}^{2,0}\left(|z|^{2} \mid \begin{array}{c}
- \\
1,0
\end{array}\right) \\
& =\frac{d^{2} z}{\pi} 2 I_{1}(2 z) K_{1}(2 z),
\end{aligned}
$$

with $K_{v}(z)$ being the modified Bessel function of the second kind [38]. Therefore, the generalized $\mathrm{SU}(1,1)$ coherent states

$$
\left|z_{E}\right\rangle_{N}=\left[\frac{|z|}{I_{1}(2|z|)}\right]^{1 / 2} \sum_{n=0}^{\infty} \frac{z^{n}}{\sqrt{n !(n+1) !}}|n\rangle
$$

form an overcomplete set in the Hilbert space $\mathscr{H}$.

The probability $\mathscr{P}_{E}(n,|z|)$ of detecting $n$ photons and the average photon number $\langle\widehat{n}\rangle_{z_{E}}$ are in this case given by the expressions

$$
\begin{aligned}
\mathscr{P}_{E}(n,|z|) & =\frac{1}{\Gamma(n+1) \Gamma(n+2)} \frac{|z|^{2 n+1}}{I_{1}(2|z|)}, \\
\langle\widehat{n}\rangle_{z_{E}} & =\frac{I_{2}(2|z|)}{I_{1}(2|z|)}|z| .
\end{aligned}
$$

In Figure 1(a) we can appreciate that the maximum of the probability $\mathscr{P}_{E}(n,|z|)$ is shifted to the right of the $|z|$-axis as $n \rightarrow \infty$. For $n=0$, the probability decreases exponentially as $|z| \rightarrow \infty$; see Figure 1(b). The latter is consistent with the behavior of the average photon number $\langle\widehat{n}\rangle_{z_{E}}$ since it grows up linearly with $|z|$; see Figure 1(c).

3.2.3. SUSY-Oscillators. The nonlinear coherent states for the supersymmetric oscillators of Sections 2.2.5 and 2.2.6 are constructed by adjusting superpositions (21) to rule (18) since the vacuum $|0\rangle$ and the 1 -photon $|1\rangle$ states are annihilated by both ladder generators, $\widehat{a}_{E}$ and $\hat{a}_{E}^{\dagger}$, just as this has been indicated in (16). They can be also obtained as a limit case from either the Hermitian approaches reported in [16, 17] (important improvements are reported in [18]) or the nonHermitian ones introduced in [20].

\section{Hilbert Spaces of Analytic Functions}

One of the main properties of the coherent states is their ability to form a basis of the Hilbert space $\mathscr{H}$, even when 


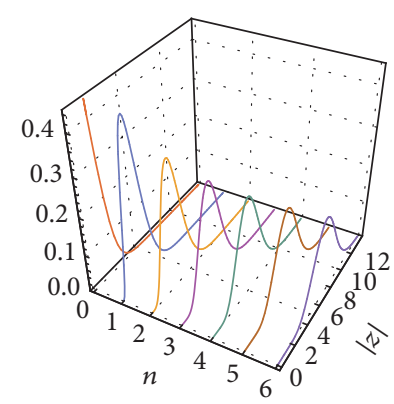

(a)

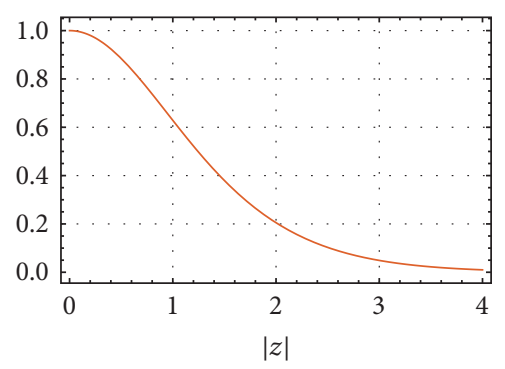

(b)

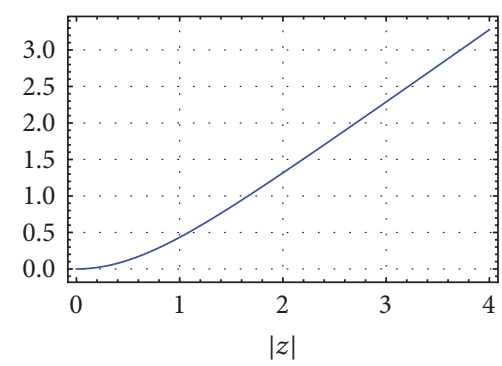

(c)

Figure 1: (a) The probability $\mathscr{P}_{E}(n,|z|)$ of detecting $n$ photons associated with the $\mathrm{SU}(1,1)$ coherent states $(34)$. (b) The probability $\mathscr{P}_{E}(0,|z|)$ of finding the system in the vacuum state as a function of $|z|$. (c) The average photon number $\langle\widehat{n}\rangle_{z_{E}}$ in terms of $|z|$.

they are nonorthogonal $[23,24]$. In this form, they can be used to represent not only any vector in $\mathscr{H}$, but also the operators defined to act on $\mathscr{H}$ in closed form [21, 22]. In the previous section we have shown that the coherent states (21) associated with the generalized oscillator algebras (2)-(3) satisfy the identity resolution (25)-(26). Here we shall go a step further by applying such property in constructing new representation spaces for the states of the new oscillators.

Using the identity resolution (25)-(26), the vectors (6) can be expressed as a superposition of coherent states

$$
|\psi\rangle=\int d \sigma_{E}(z) \psi(z)\left|z_{E}^{*}\right\rangle,
$$

where $d \sigma_{E}\left(z^{*}\right)=d \sigma_{E}(z)$, and the complex series

$$
\psi(z):=\left\langle z_{E}^{*} \mid \psi\right\rangle=\sum_{n=0}^{\infty} \frac{z^{n} \psi_{n}}{\sqrt{E(n) !}}
$$

defines the representation of $|\psi\rangle$ in the basis $\left|z_{E}\right\rangle_{N}$. As we are assuming that $E(n)$ is such that $\mathcal{N}_{E}(|z|)$ in (22) is finite, it could be shown that (37) converges for all finite $|z|$. Concrete realizations depend on the explicit form of the $E$-function. In particular, for the cases discussed in Section 3.2, $\psi(z)$ are complex-valued functions which are analytic over the whole complex $z$-plane $[17,20]$. Indeed, as these functions are holomorphic and are in one-to-one correspondence with the number eigenstates, they are elements of a Hilbert space of entire functions $\mathscr{F}_{E}$ named after Fock [39] and Bargmann [40]. In general, from the Schwarz inequality we get $|\psi(z)| \leq$ $\mathcal{N}_{E}^{-1}(z) \||\psi\rangle \|$, so that the growth of $|\psi(z)|$ will be bounded from above by the reciprocal of the normalization constant. In such a representation it follows that

$$
a_{E, o p}^{\dagger} \psi(z):=\left\langle z_{E}^{*}\left|a_{E}^{\dagger}\right| \psi\right\rangle=\sum_{n=0}^{\infty} \frac{z^{n+1} \psi_{n}}{\sqrt{E(n) !}}=z \psi(z) .
$$

Thus, the action of the creation operator $\widehat{a}_{E}^{\dagger}$ on the space $\mathscr{F}_{E}$ is reduced to the multiplication by $z$. On the other hand, for the annihilation operator one gets

$$
a_{E, o p} \psi(z):=\left\langle z_{E}^{*}\left|a_{E}\right| \psi\right\rangle=\sum_{n=1}^{\infty} \frac{z^{n-1}}{\sqrt{E(n) !}} E(n) \psi_{n} .
$$

Comparing with the derivative of $\psi(z)$ with respect to $z$,

$$
\frac{d}{d z} \psi(z)=\sum_{n=1}^{\infty} \frac{n z^{n-1}}{\sqrt{E(n) !}} \psi_{n}
$$

we realize that $\widehat{a}_{E}$ is not the canonical conjugate of $\widehat{a}_{E}^{\dagger}$ in $\mathscr{F}_{E}$ for arbitrary forms of $E$. Nevertheless, the operator $\widehat{b}_{E}$ that corresponds to (40) and satisfies $\left[\widehat{b}_{E}, \widehat{a}_{E}^{\dagger}\right]=1$ in $\mathscr{F}_{E}$ produces also the linearization of algebras (1)-(3). Preliminary results on the matter can be found in [18]; the detailed construction of $\widehat{b}_{E}$ for the general case we are dealing with will be reported elsewhere.

Of course, if $E=$ id the above expressions are reduced to those of the conventional oscillator for which the FockBargmann space $\mathscr{F}_{\text {id }}$ is formed by entire analytic functions of growth $(1 / 2,2)$. In this case, the usual boson operators $\widehat{a}_{\mathrm{id}}^{\dagger}=$ $\widehat{a}^{\dagger}$ and $\widehat{a}_{\mathrm{id}}=\widehat{a}$ correspond to the multiplication by $z$ and the derivative with respect to $z$, respectively.

The representation (37) is useful to describe pure states only. A more general and versatile representation is offered by the density operator $\widehat{\rho}$ which includes pure states, $\operatorname{Tr} \widehat{\rho}^{2}=\operatorname{Tr} \hat{\rho}$, as well as mixed states $\operatorname{Tr} \hat{\rho}^{2}<\operatorname{Tr} \hat{\rho}$. Following [21, 22], let us write $\hat{\rho}$ in $P$-representation

$$
\widehat{\rho}=\int d \sigma_{E}(z) P(z)\left|z_{E}\right\rangle\left\langle z_{E}\right| .
$$

The main point here is to find the appropriate $P$-function such that (41) can be interpreted as a "diagonal" continuous matrix representation of $\hat{\rho}$. In doing so, $P(z)$ would play the role of a nonnegative weight function, defined at all points of the complex $z$-plane.

As indicated earlier, for $E=$ id we recover the conventional coherent states $\left|z_{\text {id }}\right\rangle_{N}=|z\rangle_{N}$ of the harmonic oscillator. In such a case the density operators (41) describe the light emitted by a completely chaotic source, a model that includes all known natural light sources [23]. Also in this case the $P$-function need not have the properties of a probability distribution [22] and does not exist for all $\hat{\rho}$ [21]. However, as the classical probability theory allows for delta function distributions, $P$ can be as singular as $\delta^{(2)}(z)=$ $\delta(\operatorname{Re} z) \delta(\operatorname{Im} z)$ [21, 22]. In the quantum theory of optical 
coherence [23], if the $P$-function of a given state $\widehat{\rho}$ does not possess properties of a classical probability distribution, or it does not exist, such state does not have classical analog. Coming back to our approach, in the simplest case, the density operator $\hat{\rho}_{z}=\left|z_{E}\right\rangle\left\langle z_{E}\right|$ of any of the coherent states in (41) should be represented by a distribution $\delta^{(2)}\left(z-z^{\prime}\right)$. Otherwise, the identity (25)-(26) would be not valid. Thus, as in the conventional case, the function $P(z)$ should have, at most, $\delta$-type singularities.

To investigate the $P$-representation for other states consider the superposition

$$
|\beta\rangle=\sum_{n=0}^{\infty} \frac{\beta^{n} \sqrt{E(n) !}}{n !}|n\rangle,
$$

we may calculate the matrix elements

$$
\langle-\beta|\hat{\rho}| \beta\rangle=\int d \sigma_{E}(z) P(z) e^{\beta z^{*}-\beta^{*} z} .
$$

Then, the $P$-function is obtained from the two-dimensional inverse Fourier transform

$$
P(z)=\frac{1}{\Lambda\left(r^{2}\right)} \int d^{2} \beta\langle-\beta|\hat{\rho}| \beta\rangle e^{\beta^{*} z-\beta z^{*}} .
$$

As an immediate application consider the number eigenstate $\hat{\rho}_{n}=|n\rangle\langle n|$, then

$$
P_{n}(z)=\frac{E(n) !}{(n !)^{2} \Lambda\left(r^{2}\right)} \frac{\partial^{2 n}}{\partial z^{n} \partial z^{* n}} \delta^{(2)}(z), \quad n \geq 0 .
$$

That is, with exception of the vacuum $|0\rangle$, the $P$ representation of the number eigenstates $|n\rangle$ is as singular as the derivatives of the $\delta$-distribution. Therefore, according to [23], the fields represented by any of the number eigenstates $|n+1\rangle$ do not have classical analog. This result is quite natural since the states $|n+1\rangle$ cannot be described in classical terms, no matter the approach used in their study. In this form, the continuous matrix representation (41) is consistent with the results obtained in terms of the conventional coherent states for the vectors $|n+1\rangle$.

On the other hand, the $P$-representation of the vacuum $|0\rangle$ and the generalized coherent states $\left|z_{E}\right\rangle_{N}$ are the delta function $\delta^{(2)}\left(z-z^{\prime}\right)$, with $z^{\prime}=0$ for $|0\rangle$. The above criterion of classicality would mean that the nonlinear coherent states $\left|z_{E}\right\rangle_{N}$ are able to represent fields with classical analog, at least at the same level as the vacuum $|0\rangle$. The latter is because the singularity of the delta distribution is integrable, so that $P(z)=\delta^{(2)}(z)$ is admissible as a classical probability distribution [23]. Such statement is rather clear for $E=$ id since the conventional coherent states $\left|z_{\mathrm{id}}\right\rangle_{N}$ are indeed as classical as the vacuum $|0\rangle$. The situation changes if $E \neq \mathrm{id}$, as we are going to see in the next sections.

\section{Does the Completeness of Generalized Coherent States Imply Classicality?}

We have shown that all the nonlinear coherent states $\left|z_{E}\right\rangle_{N}$ associated with the $\ell$-order polynomials (11)-(12) have a

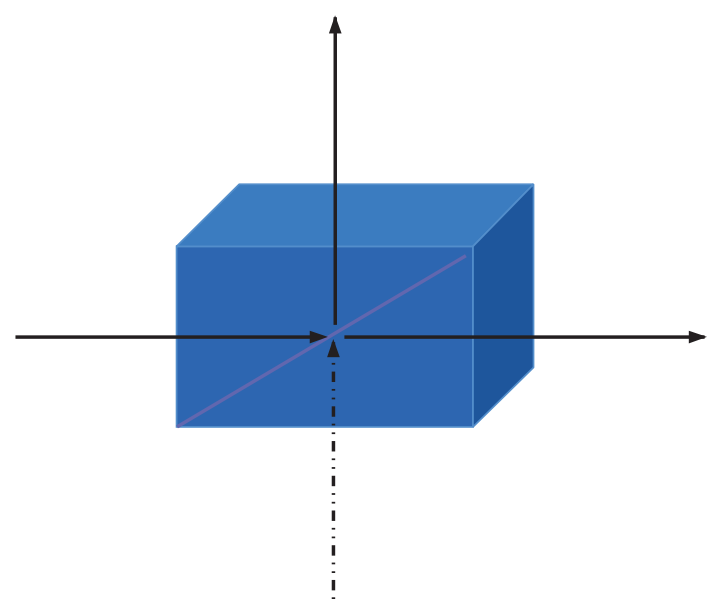

FIGURE 2: The $50: 50$ beam splitter represented by the operator BS in (46). This is a two-channel optical device operating on states $|\cdot\rangle \otimes|\cdot\rangle$, where the ket at the left (right) stands for "horizontal" ("vertical") signal. The dashed dotted arrow represents classical states like the vacuum $|0\rangle$ while the continuous arrows are associated with nonclassical states like the number eigenstates $|n+1\rangle$.

resolution to the identity (25) that is expressed in terms of the Meijer G-function (31). The latter means that the $P$ representation of all superpositions (21) is the distribution $\delta^{(2)}\left(z-z^{\prime}\right)$, just as this occurs for the vacuum $|0\rangle$ which is $P$-represented by $\delta^{(2)}(z)$. Hence, $\left|z_{E}\right\rangle_{N}$ is a displaced version of $|0\rangle$. The question is if such property is a sufficient condition for the vectors $\left|z_{E}\right\rangle_{N}$ to represent fields with classical analog. We look for an answer to this problem by using the criterion introduced in [25], as well as the parameter introduced by Mandel [4], to identify the possible classicality of such states.

5.1. Beam Splitter Criterion. Consider a single photon $|1\rangle$ entering a $50: 50$ beam splitter

$$
\mathrm{BS}=\exp \left[i \frac{\pi}{4}\left(a_{H}^{\dagger} a_{V}+a_{H} a_{V}^{\dagger}\right)\right] .
$$

The subindex " $H$ " (" $V$ ") stands for the horizontal (vertical) channel of the beam splitter; see Figure 2. As a vacuum $|0\rangle$ enters the other input port of the beam splitter, the entire input state is the product $|1\rangle \otimes|0\rangle \equiv|1,0\rangle$. Hereafter the ket at the left (right) in the tensor products $|\cdot\rangle \otimes|\cdot\rangle$ stands for "horizontal" ("vertical") signal with respect to the beam splitter shown in Figure 2. The output is the Bell state $|\beta\rangle=$ $(1 / \sqrt{2})(|1,0\rangle+i|0,1\rangle)$ which, as it is well known, encodes nonclassical correlations $[2,3]$. Such state is distinguished from the classical correlation $\rho_{\text {clas }}=(1 / 2)(|1\rangle\langle 1|\otimes| 0\rangle\langle 0|+$ $|0\rangle\langle 0|\otimes| 1\rangle\langle 1|)$ because the off-diagonal elements of its density operator $|\beta\rangle\langle\beta|$ are associated with transitions $|0\rangle \leftrightarrow|1\rangle$, occurring in both channels, that are invariant under a change of basis. That is, the off-diagonal elements $|\beta\rangle\langle\beta|-\rho_{\text {clas }}$ that are different from zero produce entanglement. In general, when 
the state $|n, 0\rangle$ enters the beam splitter, one gets the wellknown binomial distribution of bipartite photon states

$$
\mathrm{BS}|n, 0\rangle=\frac{1}{2^{n / 2}} \sum_{k=0}^{n}\left(\begin{array}{l}
n \\
k
\end{array}\right)^{1 / 2} e^{i(\pi / 2) k}|k, n-k\rangle,
$$

where a global phase has been dropped. The straightforward calculation shows that the off-diagonal elements of the density operator $\mathrm{BS}|n, 0\rangle\langle n, 0|(\mathrm{BS})^{\dagger}$ are different from zero, so that the pure state (47) encodes nonclassical correlations. These off-diagonal elements are such that measuring the number of photons at the horizontal output port of the beam splitter is affected by the result of detecting photons at the vertical port and vice versa. This last result has motivated the conjecture that the entangled output state from a beam splitter requires nonclassicality in the input state [25]. Assuming that the conjecture can be proved (see, e.g., [26]), this would be used as a criterion for nonclassicality. If the offdiagonal terms of the output state $\left|\psi_{\text {out }}\right\rangle\left\langle\psi_{\text {out }}\right|$ are nontrivial, then the input state $\left|\psi_{\text {in }}\right\rangle\left\langle\psi_{\text {in }}\right|$ is nonclassical in at least one of its two channels [25].

Let us analyze the presence of nonclassical correlations in (47) by considering the probability of finding $m$ and $r$ photons in the horizontal and vertical channels, respectively,

$$
\begin{aligned}
& |\langle m, r|\mathrm{BS}| n, 0\rangle|^{2} \\
& =\frac{\Gamma(r+1 / 2) \Gamma(m+1 / 2)}{\Gamma(r+1) \Gamma(m+1)}\left[\frac{1}{2^{r+m} B(r+1 / 2, m+1 / 2)}\right] .
\end{aligned}
$$

Here, $B(a, b)$ stands for the Euler beta function [38] which, as far as we know, cannot be expressed as $B(a, b)=f(a) g(b)$ for any functions $f$ and $g$. Therefore, probability (48) cannot be factorized as the product of two independent distributions, one for each output port of the beam splitter. This property is concomitant to the impossibility of writing the bipartite photon states (47) as the product of any state $\left|\phi_{H}\right\rangle$ of the horizontal channel with a state $\left|\phi_{V}\right\rangle$ of the vertical one, that is, $\mathrm{BS}|n, 0\rangle \neq\left|\phi_{H}\right\rangle \otimes\left|\phi_{V}\right\rangle$ if $n \neq 0$.

On the other hand, a measure of the nonclassicality of states is given by the Mandel parameter

$$
Q=\frac{(\Delta n)^{2}}{\langle\widehat{n}\rangle}-1,
$$

which indeed indicates the degree to which the statistics of a given field is sub-Poissonian [4]. For a field represented by the number eigenvector $|n+1\rangle$ one gets $Q=-1$, so that the field is sub-Poissonian $(-1 \leq Q<0)$. Classical fields like those represented by either a coherent state or a vacuum are Poissonian $(Q=0)$ or even super-Poissonian $(Q>0)$ if they correspond to thermal light.

Now, let us write $\widehat{n}_{\text {tot }}=\widehat{n}_{H}+\widehat{n}_{V}$ for the total number operator associated with either the input or the output

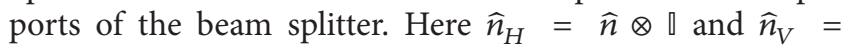
$\llbracket \otimes \hat{n}$ are the number operators for the horizontal and vertical channels, respectively. We may prove the expression $\left(\Delta n_{\text {tot }}\right)^{2}=\left(\Delta n_{H}\right)^{2}+\left(\Delta n_{V}\right)^{2}$, meaning that the variance of any signal involved with a beam splitter is the result of adding the variances of the horizontal and vertical channels. A simple calculation shows that (47) is such that $\left\langle\widehat{n}_{H}\right\rangle=\left\langle\widehat{n}_{V}\right\rangle=n / 2$ and $\left\langle\widehat{n}_{H}^{2}\right\rangle=\left\langle\widehat{n}_{V}^{2}\right\rangle=(1 / 4) n(1+n)$, so that $Q_{H}=Q_{V}=$ $-1 / 2$. Thus, the horizontal and vertical signals of (47) are subPoissonian (nonclassical).

For the present case, the conjecture indicated in [25], hereafter the (Knight) K-conjecture, is trivially verified since the number eigenstate $|n\rangle$ injected into the beam splitter to produce superposition (47) is clearly nonclassical if $n \neq 0$.

5.2. Classical Signals. Although the above discussion is true for the number eigenvectors $|n+1\rangle$ in any of the input channels, the result cannot be generalized for any superposition of such states. For instance, the input state $|z, 0\rangle$, with $\left|z_{\text {id }}\right\rangle_{N}=$ $|z\rangle_{N}$ a conventional coherent state of the harmonic oscillator, produces the separable (classical) signal

$$
\mathrm{BS}|z, 0\rangle=\left|\frac{z}{\sqrt{2}}\right\rangle_{N} \otimes\left|\frac{i z}{\sqrt{2}}\right\rangle_{N} .
$$

The latter confirms that the coherent state $|z\rangle_{N}$ is classical. The separability of the state $\mathrm{BS}|z, 0\rangle$ can be also studied in terms of the probability $\mathscr{P}(n, m,|z|)=|\langle n, m|\mathrm{BS}| z, 0\rangle|^{2}$ of detecting $n$ photons in the horizontal channel and $m$ photons in the vertical one. The result is shown in Figure 3 for $\langle\widehat{n}\rangle=16$ in the input signal. In general, the distribution spreads out on the $n m$-plane while its center is shifted along the line $m=n$ as $|z| \rightarrow \infty$; see Figure 4 .

In this case the probability $\mathscr{P}(n, m,|z|)$ can be expressed as the product of two Poisson distributions with mean value $|z|^{2} / \sqrt{2}$, one for each output port,

$$
P_{\text {oisson }}\left(\frac{|z|}{\sqrt{2}}, n\right)=\frac{e^{-|z|^{2} / 2}}{\Gamma(n+1)}\left(\frac{|z|^{2}}{2}\right)^{n} .
$$

The latter means that measuring the number of photons at the horizontal output port does not depend on the result of detecting photons at the vertical output port of the beam splitter. This result enforces the notion of classicality associated with the conventional coherent states of light.

Now, it can be shown that state (50) gives $\left(\Delta n_{\ell}\right)_{\text {out }}^{2}=$ $\left\langle\hat{n}_{\ell}\right\rangle_{\text {out }}$, with $\ell=H, V$. Then $Q_{H \text {,out }}=Q_{V \text {,out }}=0$. The latter is consistent with the separability of $\mathscr{P}(n, m,|z|)$ indicated above since the same Poisson distribution (51) determines the photon-detection for the two output ports of the beam splitter.

Summarizing the properties of the state (50), generated when the classical signal $\left|z_{\text {id }}\right\rangle_{N}$ enters a $50: 50$ beam splitter, we have the following:

(1.C) The average occupation number $\mathscr{P}(n, m,|z|)$ can be factorized as the product of two independent Poisson distributions, one for each output port.

(2.C) The variances of the input and output signals are equal.

(3.C) The variances of the horizontal and vertical output signals coincide and are equal to one-half the variance of the input signal. 


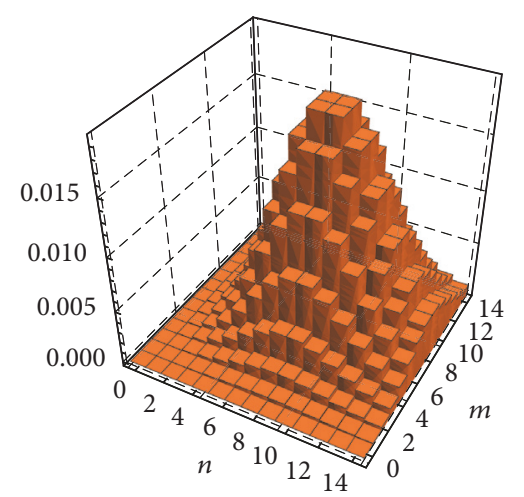

(a)

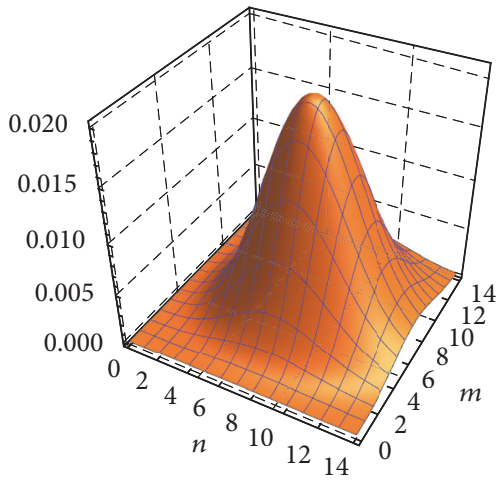

(b)

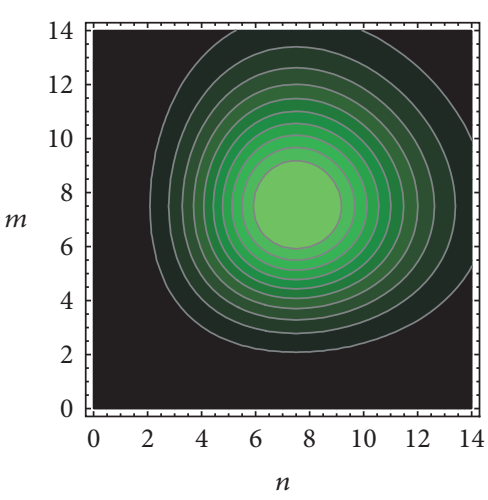

(c)

FIGURE 3: Using the classical state $|z, 0\rangle$ as input in the beam splitter of Figure 2, the probability $\mathscr{P}(n, m,|z|)$ of detecting $n$ and $m$ photons at the horizontal and vertical output ports is factorizable as the product of two independent Poisson distributions (51). The figures (a) and (b) correspond to the distribution $\mathscr{P}(n, m, 4)$ obtained for an input signal with $\langle\hat{n}\rangle=16$, and (c) shows some of its level curves, respectively.

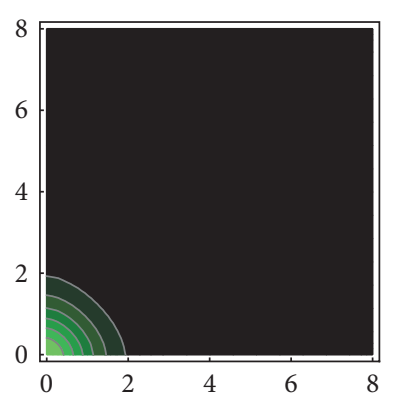

(a) $|z|=1$

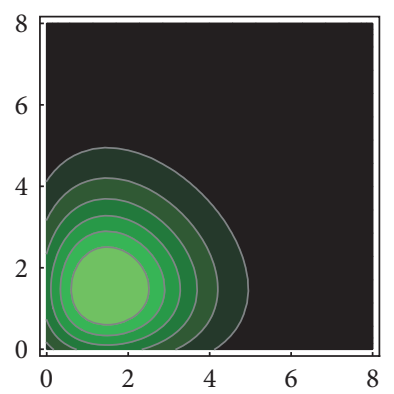

(e) $|z|=2$

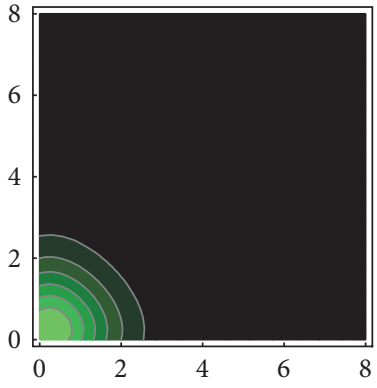

(b) $|z|=1.25$

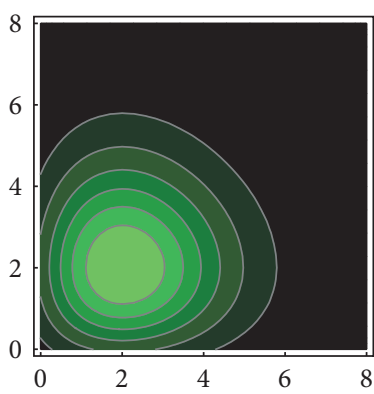

(f) $|z|=2.25$

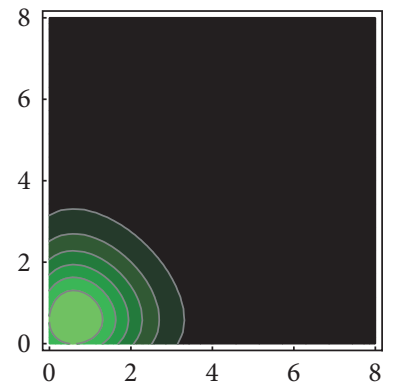

(c) $|z|=1.5$

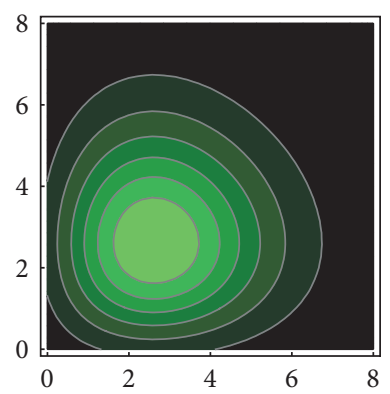

(g) $|z|=2.5$

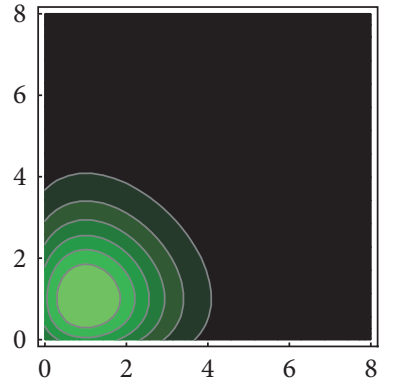

(d) $|z|=1.75$

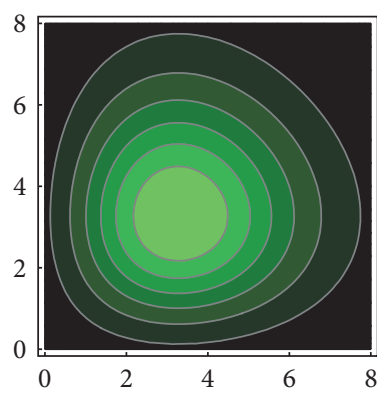

(h) $|z|=2.75$

FIgURE 4: The distribution $\mathscr{P}(n, m,|z|)$ in the $n m$-plane for the indicated values of $|z|$.

Point (1.C) and the product in (50) are concomitant since we can take one of them as a given property to verify the other one and vice versa. Here, we would like to emphasize that points (2.C) and (3.C) would serve as a criterion to investigate the possibility of factorizing the state $\mathrm{BS}|\psi, 0\rangle$, equivalently $\mathscr{P}(n, m,|z|)$, when $|\psi\rangle$ is an arbitrary superposition of photon states. As the factorization of $\mathrm{BS}|\psi, 0\rangle$ means no quantum correlations, properties (2.C) and (3.C) may imply the classicality of $|\psi\rangle$.

As we can see, also in this case the $\mathrm{K}$-conjecture is trivially verified since the conventional coherent state $\left|z_{\text {id }}\right\rangle_{N}$ injected into the beam splitter to produce the vector (50) is classical for any $|z|$.

5.3. Nonclassical Signals Associated with Generalized Oscillators. The above analysis can be extended to any state of form (21) that enters the beam splitter in the horizontal port, together with a vacuum $|0\rangle$ in the vertical channel. As we have seen, the separability of the distribution $\mathscr{P}_{E}(n, m,|z|)$ plays an important role in the identification of classicality. In the sequel we are going to pay special attention to the $\mathrm{SU}(1,1)$ coherent states derived in Section 3.2.2. The other coherent states mentioned in Section 3 lead to similar conclusions. 


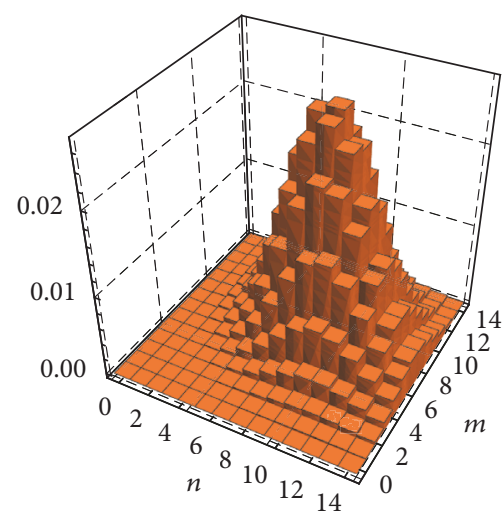

(a)

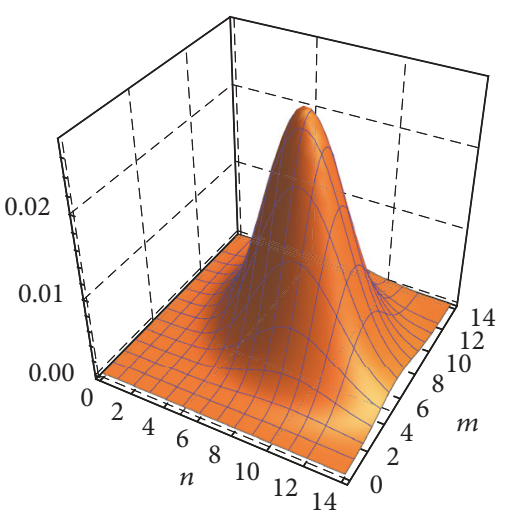

(b)

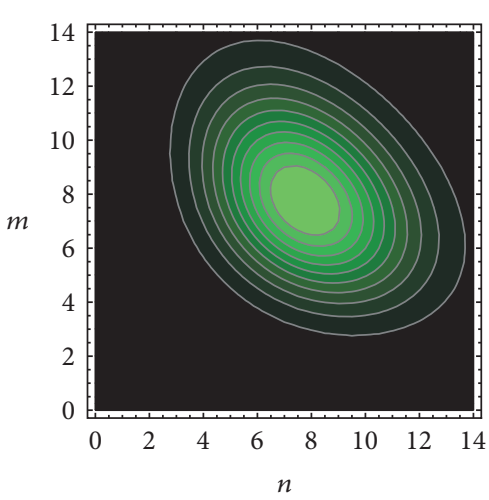

(c)

FIGURE 5: Using the input signal $\left|z_{E}, 0\right\rangle$, with $\left|z_{E}\right\rangle_{N}$, the nonlinear coherent state (34), and $\langle\hat{n}\rangle_{z_{E}}=16$, the probability $\mathscr{P}_{E}(n, m, 16.7442)$ of detecting $n$ and $m$ photons at the horizontal and vertical output ports of a beam splitter is shown in (a) and (b). Some of the corresponding level curves are depicted in (c). The average photon number $\langle\widehat{n}\rangle_{z_{E}}$ is defined in (35) as a function of $|z|$. Compare with Figure 3.

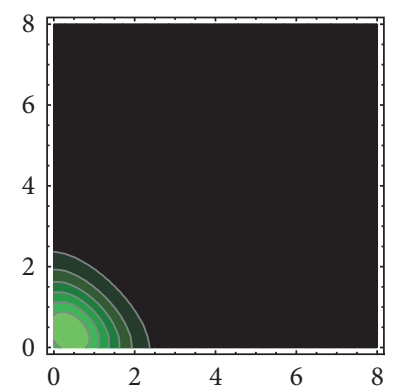

(a) $|z|=2$

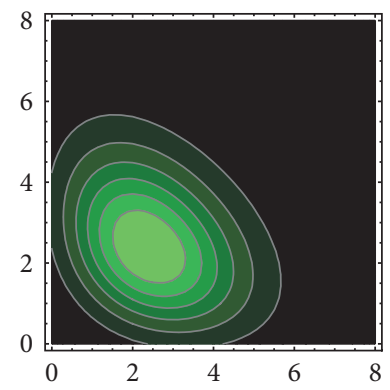

(e) $|z|=6$

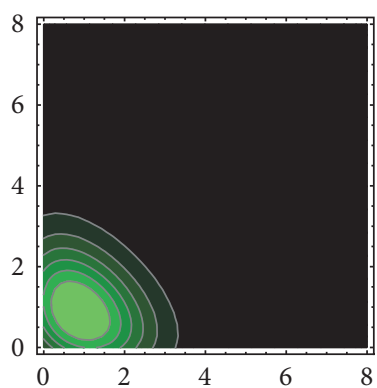

(b) $|z|=3$

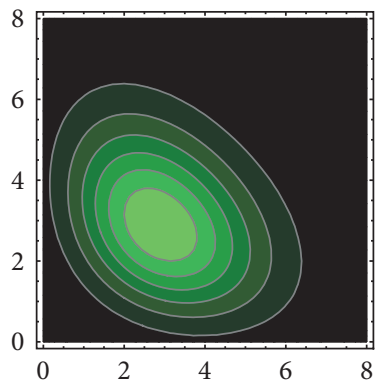

(f) $|z|=7$

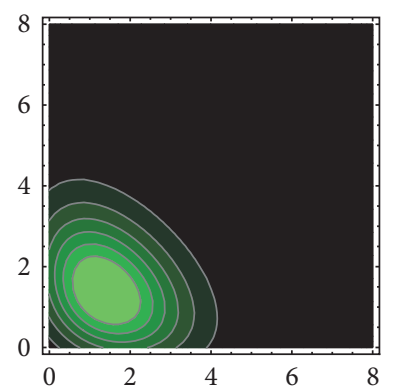

(c) $|z|=4$

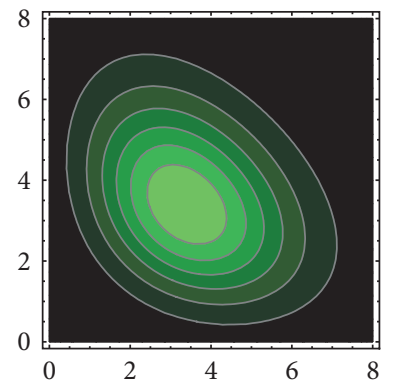

(g) $|z|=8$

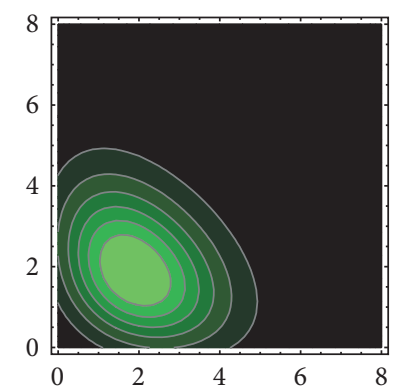

(d) $|z|=5$

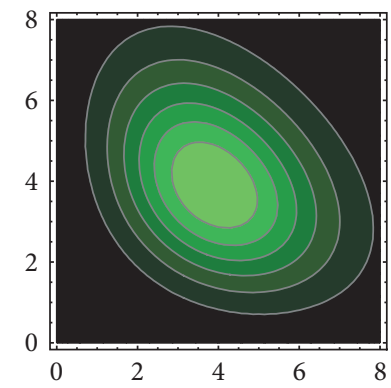

(h) $|z|=9$

Figure 6: The distribution $\mathscr{P}_{E}(n, m,|z|)$ in the $n m$-plane for the indicated values of $|z|$. Compare with Figure 4.

Using the $\mathrm{SU}(1,1)$ coherent state (34), the probability of detecting $n$ photons in the horizontal channel and $m$ photons in the vertical one gives

$$
\begin{aligned}
& \mathscr{P}_{E}(n, m,|z|) \\
& =\frac{2^{-n-m}(n+1)(m+1) B(m+1, n+1)}{\Gamma(n+1) \Gamma(n+2) \Gamma(m+1) \Gamma(m+2) I_{1}(2|z|)}|z|^{2(n+m)+1} .
\end{aligned}
$$

The behavior of this last distribution is shown in Figure 5 for the average photon number $\langle\widehat{n}\rangle_{z_{E}}=16$ in the input signal; the latter value has been chosen for comparison with the result of Figure 3. The squeezing of $\mathscr{P}_{E}(n, m,|z|)$ along the line $n=m$ is notable. Concerning the global profile, as in the previous case, distribution (52) spreads out in the $n m$-plane while its center is shifted along the line $m=n$ as $|z| \rightarrow \infty$; see Figure 6 .

As it occurred for probability (48), the presence of the beta function in (52) prohibits the factorization of $\mathscr{P}_{E}(n, m,|z|)$ as the product of two independent probability distributions if $n$ and $m$ are both different from zero. The latter means that detecting photons at the vertical output port of the beam splitter affects the counting of photons in the horizontal output port.

In turn, the variances $\left(\Delta n_{E, \text { tot }}\right)^{2}$ and $\left(\Delta n_{E, \text { tot }}\right)_{\text {out }}^{2}$ of the input $\left|z_{E}, 0\right\rangle$ and output $\mathrm{BS}\left|z_{E}, 0\right\rangle$ signals are not the same. Indeed, the latter is larger than the former for practically any $|z| \neq 0$; see Figure 7(a). However, the horizontal and 


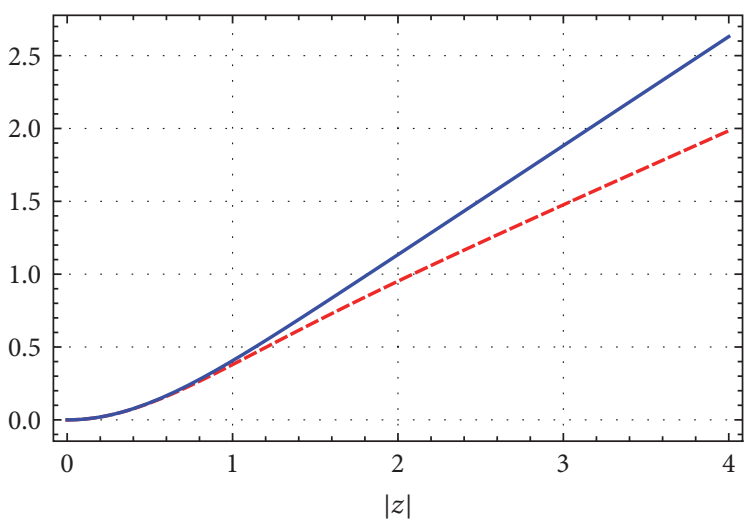

(a)

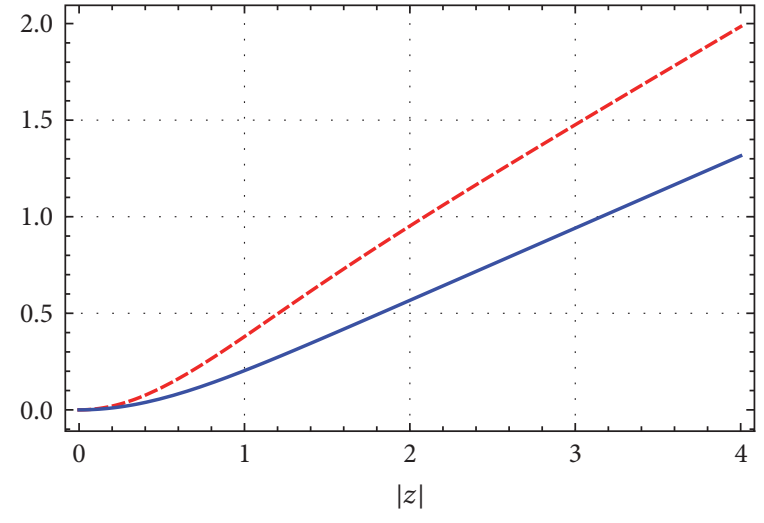

(b)

FIGURE 7: (a) The variances of the input $\left|z_{E}, 0\right\rangle$ and output $\mathrm{BS}\left|z_{E}, 0\right\rangle$ signals of a $50: 50$ beam splitter, red-dashed, and blue, respectively, with $\left|z_{E}\right\rangle_{N}$ the su(1,1) coherent state (34). (b) The variances of the signals at the horizontal and vertical output ports are equal (blue) and shorter than the variance of the input $\left|z_{E}, 0\right\rangle$ (red-dashed) for any $|z| \neq 0$.

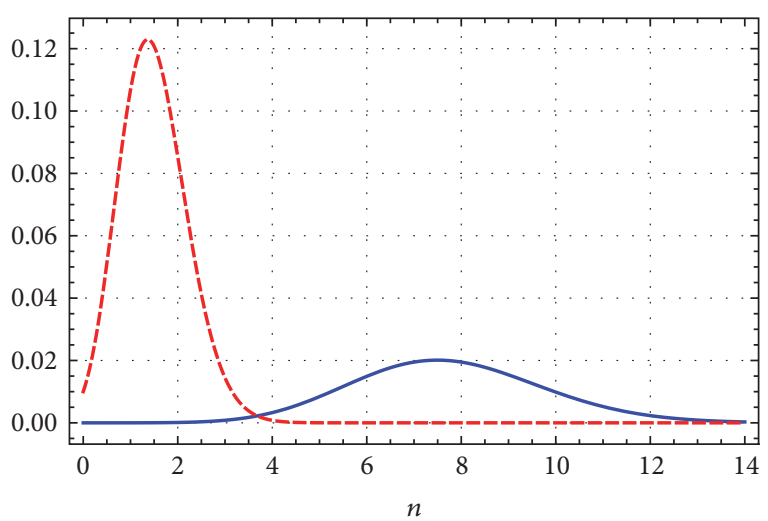

(a) $|z|=4$

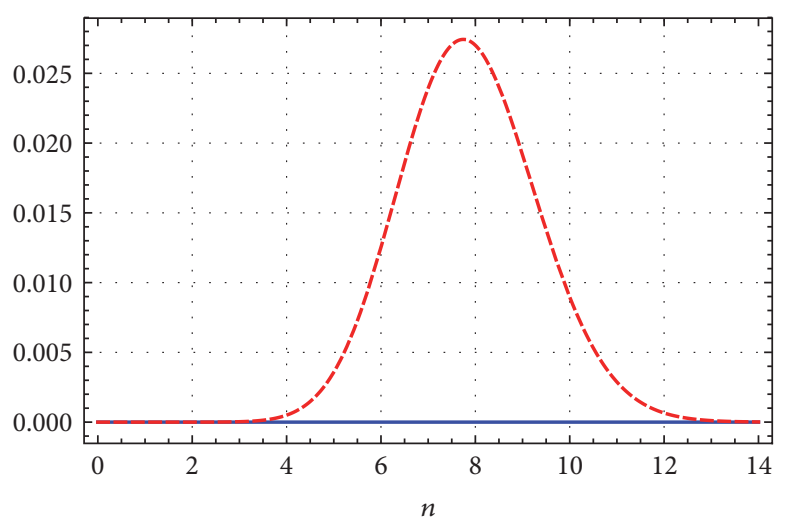

(b) $|z|=16.7442$

FIGURE 8: Squeezing along the line $n=m$ of the photon probability distributions $\mathscr{P}(n, m,|z|)$ and $\mathscr{P}_{E}(n, m,|z|)$, blue and red-dashed, respectively. In (a) the photon number averages for the incoming states $|z, 0\rangle$ and $\left|z_{E}, 0\right\rangle$ are, respectively, $\langle\hat{n}\rangle=16$ and $\langle\hat{n}\rangle_{z_{E}}=3.277$. Compare with Figure 3. In (b) we have $\langle\widehat{n}\rangle=280.369$ and $\langle\widehat{n}\rangle_{z_{E}}=16$. Compare with Figure 5. In all cases the maximum of $\mathscr{P}(n, m,|z|)$ and $\mathscr{P}_{E}(n, m,|z|)$ is reached at $\langle\widehat{n}\rangle / 2$ and $\langle\widehat{n}\rangle_{z_{E}} / 2$, respectively.

vertical output variances coincide and they are shorter than the variance of $\left|z_{E}, 0\right\rangle$; see Figure $7(\mathrm{~b})$. Such squeezing is specially clear along the line $n=m$, as this has been indicated above. See Figure 8 for a detailed comparison.

The above results indicate that the $\mathrm{SU}(1,1)$ coherent sates (34) are nonclassical although they can be interpreted as displaced versions of the vacuum $|0\rangle$. Indeed, the direct calculation shows that the Mandel parameter associated with $\left|z_{E}\right\rangle_{N}$ is such that $-1 / 2 \leq Q \leq 0$ for all $|z| \geq 0$ and $Q=0$ for $|z|=0$ only. Moreover, $Q \rightarrow-1 / 2$ as $|z| \rightarrow \infty$; see the blue curve depicted in Figure 9. That is, the statistics associated with the state (34) is sub-Poissonian, so that it is nonclassical. On the other hand, the Mandel parameter for the horizontal and vertical channels of the output signal in the interferometer gives a result that is equal to one-half the result of the input signal; see the dashed-red curve in Figure 9.
The properties of the state that is generated when the nonclassical signal $\left|z_{E}\right\rangle_{N}$ enters a 50:50 beam splitter are as follows:

(1.N) The average occupation number $\mathscr{P}_{E}(n, m,|z|)$ cannot be factorized as the product of two independent probability distributions.

(2.N) The variances of the input and output signals are different.

(3.N) The variances of the horizontal and vertical output signals are shorter than one-half the variance of the input signal.

Properties (1.N)-(3.N) are in opposition to (1.C)-(3.C) ones. As the last are not satisfied, the state $\mathrm{BS}\left|z_{E}\right\rangle$, equivalently $\mathscr{P}_{E}(n, m,|z|)$, cannot be factorized and $\left|z_{E}\right\rangle$ is nonclassical. Besides, the squeezing property (3.N) is markedly 


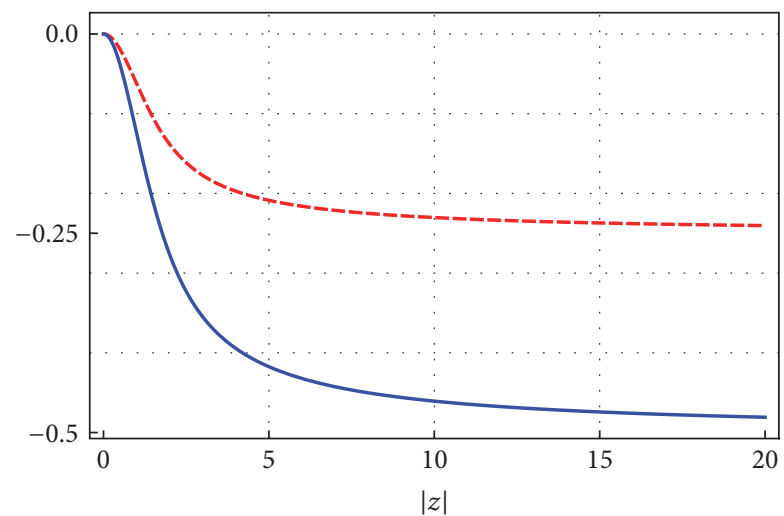

FIgURE 9: The blue curve represents the Mandel parameter $Q$ of the $\mathrm{SU}(1,1)$ coherent states $(34)$ as a function of $|z|$. This converges to $-1 / 2$ as $|z| \rightarrow \infty$. The dashed-red curve represents the Mandel parameter for both the horizontal and vertical signals at the output ports of a beam splitter when it is injected with the state $\left|z_{E}, 0\right\rangle$, where $\left|z_{E}\right\rangle_{N}$ is the coherent state (34).

different to (3.C) and implies the squeezing of the distribution $\mathscr{P}_{E}(n, m,|z|)$ along the line $n=m$.

Then, the K-conjecture is also verified for the nonlinear coherent states defined in (34). Namely, as the output state $\mathrm{BS}\left|z_{E}, 0\right\rangle$ is nonseparable, the state $\left|z_{E}\right\rangle_{N}$ in the horizontal input port of the beam splitter is nonclassical. The same conclusion is obtained for any of the nonlinear coherent states (21) that can be constructed with generalized oscillator algebras (1)-(3). However, we have shown that these states have a $P$-representation that is proportional to $\delta^{(2)}\left(z-z^{\prime}\right)$, so that they can be classified as displaced versions of the vacuum $|0\rangle$. Is there any contradiction between the nonseparability of the states $\mathrm{BS}\left|z_{E}, 0\right\rangle$ and the $P$-representation of $\left|z_{E}\right\rangle_{N}$ ?

5.4. Refinement of the Criterion. To clarify the results of the previous sections let us emphasize that the states $\mathrm{BS}|n, 0\rangle$ defined in (47) are nothing but a class of generalized coherent states [41] (see also [42]) associated with the su(2) Lie algebra realized in terms of two oscillators, that is, in the so-called Schwinger representation [43],

$$
|\xi\rangle=\frac{1}{\left(1+|\xi|^{2}\right)^{-n / 2}} \sum_{k=0}^{n}\left(\begin{array}{l}
n \\
k
\end{array}\right)^{1 / 2} \xi^{k}|k, n-k\rangle .
$$

A simple inspection shows that making $\xi=e^{i(\pi / 2)}$ in this last expression gives state (47). The states $|\xi\rangle$ also have a resolution to the identity and are represented by a $P$-function that is proportional to the $\delta$-distribution. However, they are nonclassical, as this has been discussed in Section 5.1.

In many ways, the action of the beam splitter on the incoming state $|n, 0\rangle$ is equivalent to a double-slit interference experiment in the single-photon regime; see, for example, $[27,28]$. Indeed, since the detector and electronic instrumentation dead-time limitations make the direct measurement of antibunching in a double-slit experiment difficult [28], the output intensities are instead measured by using a $50: 50$ beam splitter and two detectors [27]. These and other photon correlation experiments have their origin in the HanburyBrown and Twiss experiments [29], the results of which have shown the importance of distinguishing between the first two orders of coherence. Following Glauber [23], given the normalized form of the correlation functions

$$
\begin{aligned}
& g^{(n)}\left(x_{1}, \ldots, x_{2 n}\right)=\frac{G^{(n)}\left(x_{1}, \ldots, x_{n}\right)}{\prod_{j=1}^{2 n}\left\{G^{(1)}\left(x_{j}, x_{j}\right)\right\}^{1 / 2}}, \\
& x_{j} \equiv\left(\vec{r}_{j}, t_{j}\right),
\end{aligned}
$$

the necessary condition of coherence is that $\left|g^{(j)}\right|=1$. The first-order coherence is obtained if $j=1$. This coincides with the definition of coherence used in optics previous to the theory of Glauber. On the other hand, a field characterized by $n$th order coherence corresponds to $j \leq n$. The full coherence implies that $\left|g^{(n)}\right|=1$ holds for all $n$. Light beams from ordinary sources can be made optimally firstorder coherent but they lack second-order coherence [23]. In turn, the conventional coherent states are coherent to all orders. Next, we follow [23] and adopt the factorization of $G^{(n)}$ producing $g^{(n)}=1$ in (54) as the definition of the $n$th order coherent fields.

The straightforward calculation shows that the horizontal and vertical channels of the nonlinear coherent states (47), equivalently (53), lack second-order coherence since $g^{(2)}=$ $1-1 / n<1$ for $n \neq 0$. This means that the photons in such ports are anticorrelated, so that they cannot be created or annihilated simultaneously. The result $g^{(2)} \neq$ 1 is due to the fact that $G^{(2)}$ cannot be factorized as the product of two $G^{(1)}$-functions. The statement is equivalent to the impossibility of factorizing either the vector BS $|n, 0\rangle$ or probability (48). Notice however that $g^{(2)} \rightarrow 1$ for $n \rightarrow$ $\infty$. That is, for a large number of photons, state (47) can be associated with the results of an interference experiment involving a classical field.

As regards the $\mathrm{SU}(1,1)$ coherent states (34), for the horizontal and vertical output ports of the beam splitter we obtain the expression

$$
g^{(2)}=\frac{I_{1}(2|z|) I_{3}(2|z|)}{I_{2}^{2}(2|z|)} .
$$

This last function satisfies $g^{(2)}<1$ for all finite $|z|$ and goes to one for $|z| \rightarrow \infty$; see Figure 10. As $|z| \gg 1$ implies a large average photon number; see Figure 1(c), the latter result means that the coherent states (34) allow for bunching of photons only at the limit $\langle\widehat{n}\rangle_{z_{E}} \rightarrow \infty$. Again, the result $g^{(2)} \neq$ 1 is associated with the fact that neither the function $G^{(2)}$ nor probability (52) nor the state $\mathrm{BS}\left|z_{E}, 0\right\rangle$ can be factorized.

As we have seen, the nonlinear coherent states $\left|z_{E}\right\rangle_{N}$ are not full coherent although their $P$-representation is a delta function, so that they cannot be considered "classical" in the sense established by Glauber [23]. Besides, the impossibility of factorizing either the second-order correlation function 


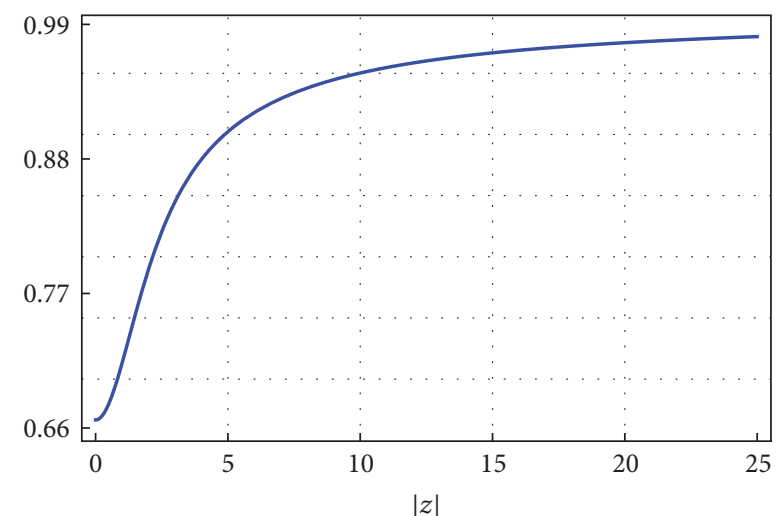

FIGURE 10: The normalized form of the correlation function of second order (54) for the horizontal and vertical output channels of a $50: 50$ beam splitter injected by one of the $\mathrm{SU}(1,1)$ coherent states $\left|z_{E}\right\rangle$ defined in (34).

$G^{(2)}$, the probability of detecting $n$ and $m$ photons in the horizontal and vertical output channels, or the output state $\mathrm{BS}\left|z_{E}, n\right\rangle$ associated with a 50:50 beam splitter means that the fields represented by $\left|z_{E}\right\rangle_{N}$ are nonclassical.

\section{Concluding Remarks}

We have shown that the nonlinear coherent states associated with a series of generalized oscillator algebras can be written in the same mathematical form. If such algebras are polynomial the related coherent states satisfy a closure relation that is uniquely expressed in terms of the Meijer $G$-function. We have also shown that although the $P$-representation of these states is as singular as the delta function, they have no classical analog. The latter is due to the fact that such states are not full coherent in the sense established by Glauber in his quantum theory of optical coherence [23]. Then, a field represented by any nonlinear coherent state which is $P$-represented by a delta function will have classical analog whenever such state is full coherent.

As a byproduct of this work, we have shown that the criterion of separability introduced in [25] for distinguishing nonclassicality of states can be refined by considering also the separability of either the second-order correlation function $G^{(2)}$ or the probability of detecting $n$ and $m$ photons in the horizontal and vertical output ports of a $50: 50$ beam splitter.

\section{Appendix}

\section{The E-Exponential Function}

The E-function introduced in Section 3,

$$
\begin{aligned}
e_{E}^{x}=\sum_{n=0}^{\infty} \frac{x^{n}}{E(n) !}, & \\
& E(n) !=E(1) E(2) \cdots E(n), E(0) ! \equiv 1,
\end{aligned}
$$

acquires a simple form if $E(n)$ is the polynomial of degree $\ell$ defined in (11)-(12). Explicitly,

$$
\begin{aligned}
e_{E}^{x}={ }_{1} F_{\ell}\left(1 ; 1+\delta_{j} ; \frac{x}{\gamma_{\ell}}\right), & \\
\delta_{j} & =\frac{\beta_{j}}{\alpha_{j}}, \gamma_{\ell}=\alpha_{1} \cdots \alpha_{\ell}, j=1, \ldots, \ell,
\end{aligned}
$$

where

$$
\begin{aligned}
& { }_{p} F_{q}\left(a_{1}, \ldots, a_{p}, b_{1}, \ldots, b_{q} ; z\right) \equiv{ }_{p} F_{q}\left(a_{j}, b_{j} ; z\right) \\
& =\frac{\Gamma\left(b_{1}\right) \cdots \Gamma\left(b_{q}\right)}{\Gamma\left(a_{1}\right) \cdots \Gamma\left(a_{p}\right)} \sum_{n=0}^{\infty} \frac{\Gamma\left(a_{1}+n\right) \cdots \Gamma\left(a_{p}+n\right)}{\Gamma\left(b_{1}+n\right) \cdots \Gamma\left(b_{q}+n\right)} \frac{z^{n}}{n !}
\end{aligned}
$$

stands for the generalized hypergeometric function [38]. The following particular cases are of special interest:

(i) For $\ell=1$ and $\beta \neq 0$ we have

$$
\begin{aligned}
e_{E}^{x} & ={ }_{1} F_{1}\left(1,1+\delta, \frac{x}{\alpha}\right) \\
& =\delta e^{x / \alpha}\left(\frac{\alpha}{x}\right)^{\delta}\left[\Gamma(\delta)-\Gamma\left(\delta, \frac{x}{\alpha}\right)\right] .
\end{aligned}
$$

In particular, for $\beta=0$ we get $\delta=0$ and

$$
e_{E}^{x}=\lim _{\delta \rightarrow 0}{ }_{1} F_{1}\left(1,1+\delta, \frac{x}{\alpha}\right)=e^{x / \alpha} .
$$

(ii) For $\ell=2$ and either $\beta_{1}=0$ or $\beta_{2}=0$, one gets

$$
\begin{aligned}
e_{E}^{x} & ={ }_{1} F_{2}(1 ; 1,1+\delta ; x)={ }_{1} F_{2}(1 ; 1+\delta, 1 ; x) \\
& =\Gamma(1+\delta)\left(\frac{\gamma_{2}}{x}\right)^{\delta / 2} I_{\delta}\left(2 \frac{x}{\gamma_{2}}\right),
\end{aligned}
$$

with $I_{\nu}(z)$ the modified Bessel function of the first kind [38].

(iii) For $\ell=3$ and $\alpha_{1}=\alpha_{2}=\alpha_{3}=\beta_{1}=\alpha$, with arbitrary $\beta_{2}$ and $\beta_{3}$, the $E$-exponential function (A.1) becomes

$$
\begin{aligned}
e_{E}^{x} & ={ }_{1} F_{3}\left(1 ; 2,1+\delta_{2}, 1+\delta_{3} ; \frac{x}{\gamma_{3}}\right) \\
& =\frac{\gamma_{3} \delta_{2} \delta_{3}}{x}\left[{ }_{0} F_{2}\left(\delta_{2}, \delta_{3} ; \frac{x}{\gamma_{3}}\right)-1\right] .
\end{aligned}
$$

\section{Conflicts of Interest}

The authors declare that they have no conflicts of interest.

\section{Acknowledgments}

The authors acknowledge the financial support from Instituto Politécnico Nacional, Mexico (Project SIP20170233), the Spanish MINECO (Project MTM2014-57129-C2-1-P), and Junta de Castilla y León (VA057U16). Kevin Zelaya and Zurika Blanco-Garcia gratefully acknowledge the funding received through the CONACyT scholarships 45454 and 489856, respectively. 


\section{References}

[1] V. V. Dodonov and V. I. Man'ko, Eds., Theory of Nonclassical States of Light, Taylor \& Francis, New York, NY, USA, 2003.

[2] D. Bouwmeester, A. Ekert, and A. Zeilinger, The Physics of Quantum Information: Quantum Cryptography, Quantum Teleportation, Quantum Computation, Springer, New York, NY, USA, 2000.

[3] M. P. Silverman, Quantum Superposition: Counterintuitive Consequences of Coherence, Entanglement, and Interferece, SpringerVerlag, Berlin, 2008.

[4] L. Mandel, "Sub-Poissonian photon statistics in resonance fluorescence," Optics Expresss, vol. 4, no. 7, pp. 205-207, 1979.

[5] D. F. Walls, "Squeezed states of light," Nature, vol. 306, no. 5939, pp. 141-146, 1983.

[6] L. C. Biedenharn, "The quantum group $\mathrm{SUq}(2)$ and a qanalogue of the boson operators," Journal of Physics A: Mathematical and General, vol. 22, no. 18, pp. L873-L878, 1989.

[7] A. J. Macfarlane, "On q-analogues of the quantum harmonic oscillator and the quantum group SU(2)q," Journal of Physics A: Mathematical and General, vol. 22, no. 21, pp. 4581-4588, 1989.

[8] A. I. Solomon, "A characteristic functional for deformed photon phenomenology," Physics Letters A, vol. 196, no. 1-2, pp. 29-34, 1994.

[9] R. L. de Matos Filho and W. Vogel, "Nonlinear coherent states," Physical Review A: Atomic, Molecular and Optical Physics, vol. 54, no. 5, pp. 4560-4563, 1996.

[10] V. I. Man’ko, G. Marmo, E. C. G. Sudarshan, and F. Zaccaria, "foscillators and nonlinear coherent states," Physica Scripta, vol. 55, no. 5, pp. 528-541, 1997.

[11] B. Mielnik and O. Rosas-Ortiz, "Factorization: little or great algorithm?" Journal of Physics A: Mathematical and General, vol. 37, no. 43, pp. 10007-10035, 2004.

[12] A. A. Andrianov and M. V. Ioffe, "Nonlinear supersymmetric quantum mechanics: concepts and realizations," Journal of Physics A: Mathematical and General, vol. 45, no. 50, Article ID 503001, 2012.

[13] H. Aoyama, M. Sato, and T. Tanaka, "General forms of a N-fold supersymmetric family," Physics Letters B, vol. 503, no. 3-4, pp. 423-429, 2001.

[14] H. Aoyama, M. Sato, and T. Tanaka, "N-fold supersymmetry in quantum mechanics: general formalism," Nuclear Physics B, vol. 619, no. 1-3, pp. 105-127, 2001.

[15] B. Mielnik, "Factorization method and new potentials with the oscillator spectrum," Journal of Mathematical Physics, vol. 25, no. 12, pp. 3387-3389, 1984.

[16] D. J. Fernández, L. M. Nieto, and O. Rosas-Ortiz, "Distorted Heisenberg algebra and coherent states for isospectral oscillator hamiltonians," Journal of Physics A: Mathematical and General, vol. 28, no. 9, pp. 2693-2708, 1995.

[17] J. O. Rosas-Ortiz, "Fock-Bargmann representation of the distorted Heisenberg algebra," Journal of Physics A: Mathematical and General, vol. 29, no. 12, pp. 3281-3288, 1996.

[18] D. J. Fernández, V. Hussin, and O. Rosas-Ortiz, "Coherent states for Hamiltonians generated by supersymmetry," Journal of Physics A: Mathematical and General, vol. 40, no. 24, pp. 64916511, 2007.

[19] O. Rosas-Ortiz, O. Castaños, and D. Schuch, "New supersymmetry-generated complex potentials with real spectra," Journal of Physics A: Mathematical and Theoretical, vol. 48, no. 44, Article ID 445302, 2015.
[20] O. Rosas-Ortiz and K. Zelaya, "Bi-orthogonal approach to non-Hermitian Hamiltonians with the oscillator spectrum: generalized coherent states for nonlinear algebras," Annals of Physics, vol. 388, pp. 26-53, 2018.

[21] R. J. Glauber, "Coherent and incoherent states of the radiation field," Physical Review A: Atomic, Molecular and Optical Physics, vol. 131, no. 6, pp. 2766-2788, 1963.

[22] E. C. Sudarshan, "Equivalence of semiclassical and quantum mechanical descriptions of statistical light beams," Physical Review Letters, vol. 10, pp. 277-279, 1963.

[23] R. J. Glauber, Quantum Theory of Optical Coherence: Selected Papers and Lectures, Wiley-VCH, Weinheim, 2007.

[24] J. R. Klauder and E. C. Sudarshan, Fundamentals of Quantum Optics, W. A. Benjamin Inc., New York, NY, USA, 1968.

[25] M. S. Kim, W. Son, V. Bužek, and P. L. Knight, "Entanglement by a beam splitter: nonclassicality as a prerequisite for entanglement," Physical Review A: Atomic, Molecular and Optical Physics, vol. 65, no. 3, Article ID 032323, 2002.

[26] X.-b. Wang, “Theorem for the beam-splitter entangler," Physical Review A: Atomic, Molecular and Optical Physics, vol. 66, no. 2, Article ID 024303, 2002.

[27] P. Grangier, G. Roger, and A. Aspect, "Experimental evidence for a photon anticorrelation effect on a beam splitter: a new light on single-photon interferences," EPL (Europhysics Letters), vol. 1, no. 4, pp. 173-179, 1986.

[28] W. Rueckner and J. Peidle, "Young's double-slit experiment with single photons and quantum eraser," American Journal of Physics, vol. 81, no. 12, Article ID 1.4819882, 2013.

[29] R. H. Brown and R. Q. Twiss, "Correlation between photons in two coherent beams of light," Nature, vol. 177, pp. 27-29, 1956.

[30] E. T. Jaynes and F. W. Cummings, "Comparison of Quantum and Semiclassical Radiation Theories with Application to the Beam Maser," Proceedings of the IEEE, vol. 51, no. 1, pp. 89-109, 1963.

[31] B. Buck and C. V. Sukumar, "Exactly soluble model of atomphonon coupling showing periodic decay and revival," Physics Letters A, vol. 81, no. 2-3, pp. 132-135, 1981.

[32] C. V. Sukumar and B. Buck, "Multi-phonon generalisation of the Jaynes-Cummings model," Physics Letters A, vol. 83, no. 5, pp. 211-213, 1981.

[33] F. H. Jackson, “On q-Definite Integrals," Q. J. Pure Appl. Math, vol. 41, no. 193, 1910.

[34] J. Bertrand, P. Bertrand, and J. Ovarlez, "The Mellin Transform," in The transforms and applications handbook, A. D. Poularikas, Ed., CRC Press, Boca Raton, 2000.

[35] O. de los Santos-Sanchez and J. Recamier, "The f-deformed Jaynes-Cummings model and its nonlinear coherent states," Journal of Physics A: Mathematical and General, vol. 45, Article ID 015502, 2012.

[36] M. Arik and D. D. Coon, "Hilbert spaces of analytic functions and generalized coherent states," Journal of Mathematical Physics, vol. 17, no. 4, pp. 524-527, 1976.

[37] A. Jannussis, G. Bbodimas, D. Soublas, and V. Zisis, "Remarks on the q-quantization," Lettere Al Nuovo Cimento, vol. 30, no. 4, pp. 123-127, 1981.

[38] F. W. J. Olver, D. W. Lozier, R. F. Boisvert, and C. W. Clark, Eds., NIST Handbook of Mathematical Functions, Cambridge University Press, Cambridge, UK, 2010.

[39] V. Fock, "Verallgemeinerung und Lösung der Diracschen statistischen Gleichung," Zeitschrift für Physik, vol. 49, no. 5-6, pp. 339-357, 1928. 
[40] V. Bargmann, "On a Hilbert space of analytic functions and an associated integral transform," Communications on Pure and Applied Mathematics, vol. 14, pp. 187-214, 1961.

[41] A. M. Perelomov, Generalized Coherent States and Their Applications, Texts and Monographs in Physics, Springer-Verlag, Heidelberg, Germany, 1986.

[42] A. O. Barut and L. Girardello, "New 'coherent' states associated with non-compact groups," Communications in Mathematical Physics, vol. 21, pp. 41-55, 1971.

[43] J. Schwinger, "On Angular Momentum," in Quantum Theory of Angular Momentum, L. C. Biedenharn and H. Van Dam, Eds., Academic, New York, NY, USA, 1965. 


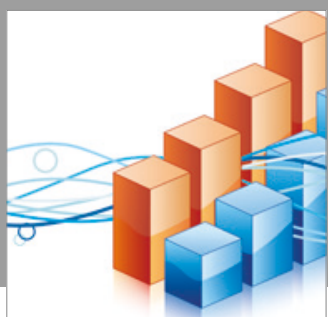

Advances in

Operations Research

vatersals

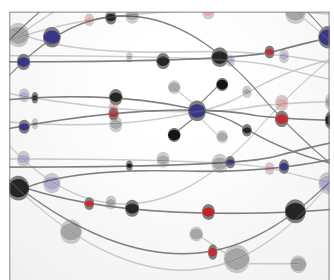

\section{The Scientific} World Journal
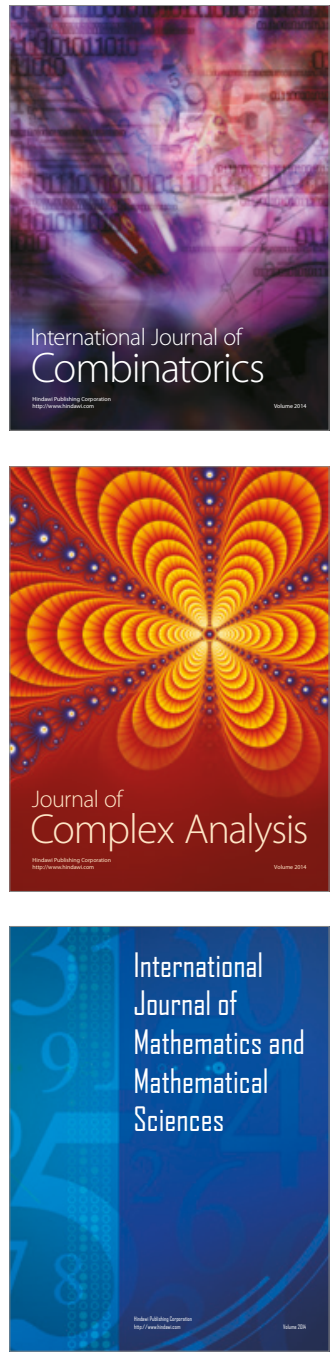
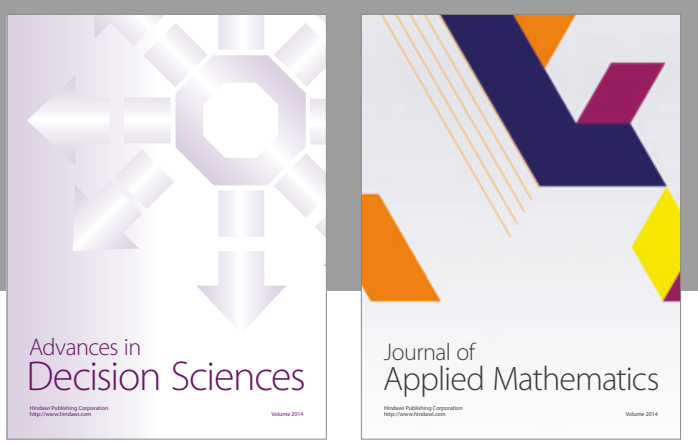

Algebra

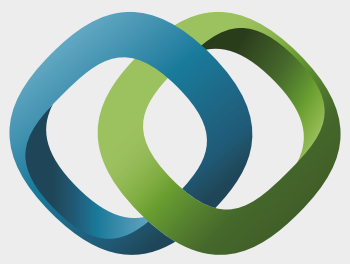

\section{Hindawi}

Submit your manuscripts at

https://www.hindawi.com
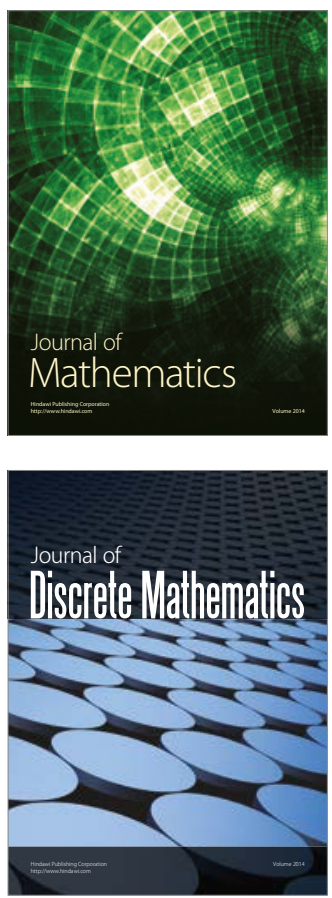

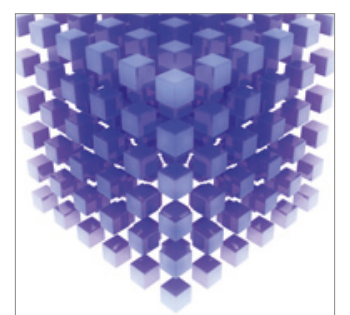

Mathematical Problems in Engineering
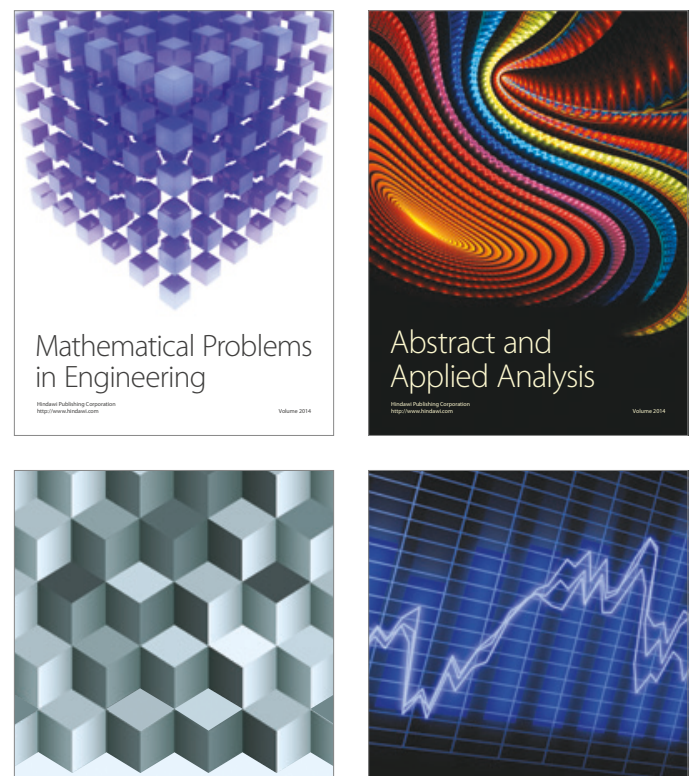

Journal of

Function Spaces

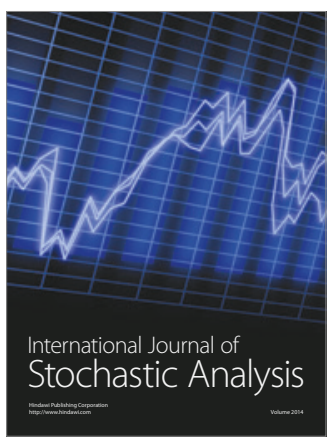

Probability and Statistics
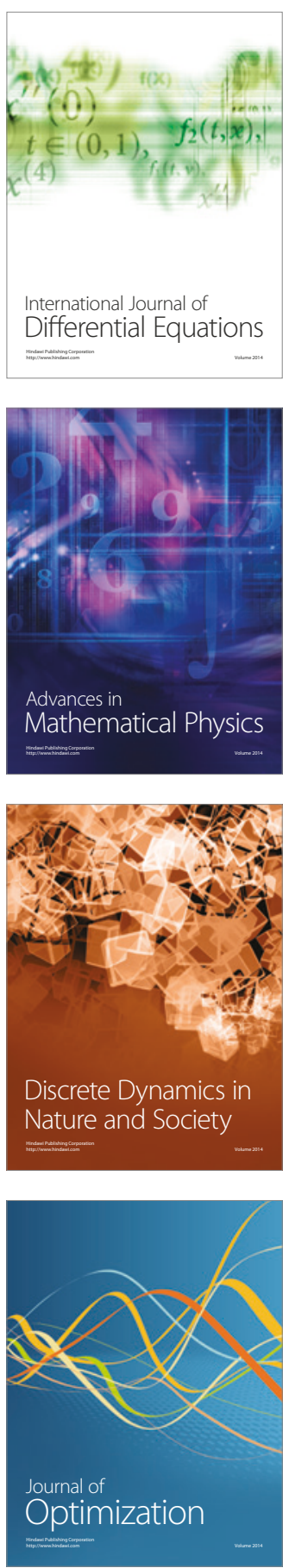